Preprints of the

Max Planck Institute for

Research on Collective Goods

Bonn 2007/8

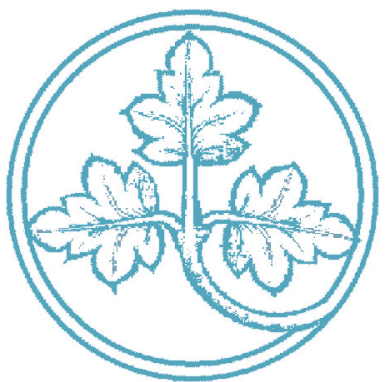

A Reconsideration of the Jensen-Meckling Model of Outside Finance

Martin Hellwig

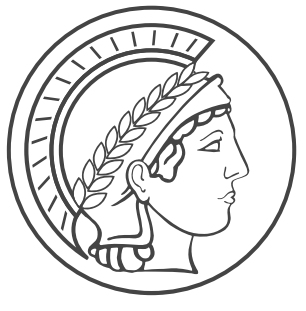




\title{
A Reconsideration of the Jensen-Meckling Model of Outside Finance
}

\author{
Martin Hellwig
}

May 2007 


\title{
A Reconsideration of the Jensen-Meckling Model of Outside Finance*
}

\author{
Martin F. Hellwig \\ Max Planck Institute for Research on Collective Goods \\ Kurt Schumacher-Str. 10 \\ D - 53113 Bonn, Germany \\ hellwig@coll.mpg.de
}

May 23, 2007

\begin{abstract}
The paper studies outside finance in a model of two-dimensional moral hazard, involving risk choices as well as effort choices. If the entrepreneur has insufficient funds, a first-best outcome cannot be implemented. Second-best outcomes involve greater failure risk than firstbest outcomes. For a Cobb-Douglas technology, second-best effort and investment levels are smaller than first-best; for other technologies, they depend on the elasticity of substitution. If firm returns not too noisy signals of behaviour, suitable incentives can be provided by some mix of debt and equity issues. If firm returns involve too much noise, this is not possible.

Key Words: Financial Contracting, Debt Finance, Equity Finance, Moral Hazard, RiskChoices.

JEL Classification: D86, G30, G32.
\end{abstract}

\section{Introduction}

This paper takes a new look at the approach to outside finance that was developed by Jensen and Meckling (1976) some thirty years ago. Their

${ }^{*}$ This revision of Hellwig (1994) was prepared for the ECGI-JFI-CFS-Mannheim conference on Financial Contracting. Without implicating them, I thank Helmut Bester, Patrick Bolton, Margaret Bray, Christoph Engel, Mike Fishman, Thomas Gehrig, Mark Hahmeier, Andreas Nicklisch, Jean Tirole, and Elu von Thadden for helpful discussions and comments. I am also grateful for financial support of the original research by the Scheizerischer Nationalfonds. 
approach is summarized by the following propositions:

- Outside finance involves "agency costs" because relations between, on the one hand, financiers and, on the other hand, entrepreneurs or managers are affected by moral hazard.

- Different forms of outside finance involve different forms of moral hazard and, therefore, different types of agency costs. Outside equity finance is mainly affected by moral hazard with respect to the level of effort that the manager exerts. Outside debt finance is mainly affected by moral hazard concerning the risks inherent in the entrepreneur's strategy.

- The equilibrium capital structure of the firm minimizes the sum of all agency costs. To the extent that monitoring and bonding activities affect moral hazard as well, the equilibrium capital structure is determined jointly with these activities so as to minimize the sum of all agency, monitoring, and bonding costs.

Beyond the narrow issue of how to explain capital structure, the work of Jensen and Meckling has initiated the general research program to explain observed financing patterns and financial institutions in terms of optimal ( $n$ th best) responses of market participants to problems of moral hazard and incomplete information. This research program has shaped the entire literature. ${ }^{1}$ In the nineties, the direction of research has shifted from "complete" to "incomplete" contracts, focussing on control rights assignments, rather than return patterns associated with different securities. ${ }^{2}$ However, the incomplete-contracts approach also follows the overall program of explaining observed financing patterns as optimal solutions to contracting problems with moral hazard and incomplete information.

This paper returns to the original Jensen-Meckling problem of explaining capital structure in terms of the incentive implications of return patterns associated with different mixes of instruments for outside finance. I am not trying to suggest that control rights are unimportant. However, the incentive implications of different return patterns are also important, even for the incomplete-contracts approach. Thus, Dewatripont and Tirole (1994) refer to these incentive implications when they study why control rights assignments and return patterns tend to be linked the way they are for debt and equity. They rely on the original insights of Jensen and Meckling

\footnotetext{
${ }^{1}$ For an early survey, see Harris and Raviv (1991).

${ }^{2}$ See, e.g., Aghion and Bolton $(1989,1992)$ or Hart $(1993,1995)$.
} 
that outside equity finance is more susceptible to moral hazard concerning effort choices and outside debt finance is more susceptible to moral hazard concerning risk choices.

Jensen and Meckling did not actually provide the encompassing analysis that their conclusions would seem to have required. They provided piecemeal analyses of (i) the incentive effects of outside equity finance when moral hazard concerns effort choices only and (ii) the incentive effects of outside debt finance when moral hazard concerns risk choices only. They did not put the different pieces of the puzzle together in a model with both sources of moral hazard at the same time. This is where the present paper steps in.

The paper develops an integrated model with moral hazard that involves effort choices and risk choices at the same time. ${ }^{3}$ It turns out that there is a natural interdependence between these two sources of moral hazard. The moral-hazard problem as a whole takes on an extra dimension if the entrepreneur is able to conceal a low effort choice behind a relatively high return realization made possible by a high risk choice. Separate, piecemeal analyses of the different types of moral hazard by themselves do not capture this extra dimension. It is therefore inappropriate to think of the total agency cost that is associated with a given mix of debt and equity finance as a sum of the agency costs of the debt issue and the agency costs of the equity issue, one concerning risk choices and the other concerning effort choices. In the integrated model, risk choices and effort choices are two sides of the same coin. It makes no sense to talk about their agency costs separately.

It also makes no sense to talk separately about the agency costs of debt instruments and the agency costs of equity instruments. Outside equity finance and outside debt finance jointly determine the total investment that can be financed and the overall incentive scheme that the entrepreneur or manager faces as he takes his effort-and-risk choices. One must think comprehensively in terms of overall incentive effects of a given package of financial instruments on the combination of effort and risk levels that is chosen.

Indeed, it is not at all clear that an optimal incentive scheme for the given moral-hazard problem should take a form that can be interpreted in terms of standard financial instruments. Standard financial instruments induce incentive schemes that have a special mathematical structure, making the entrepreneur's income a piecewise linear, continuous function of realized

\footnotetext{
${ }^{3}$ A rudimentary form of the model was already used in Bester and Hellwig (1987). The model here is more general and therefore more suitable for dealing with the issues raised by Jensen and Meckling.
} 
overall returns. Why should a scheme with such a structure be optimal in dealing with the given incentive problem? Standard incentive theory often comes up with much more complicated, highly nonlinear, somtimes even discontinuous incentive schemes. ${ }^{4}$

Following most of the literature on financial contracting, the paper assumes that all parties are risk neutral. Risk sharing considerations play no role. Incentive considerations arise because of limitations to the entrepreneur's ability to pay, more precisely, because, in each state of the world, the entrepreneur's ability to repay his financiers depends on the return realization in that state; this return realization in turn depends on the entrepreneur's prior risk and effort choices.

Under risk neutrality, the integrated moral-hazard problem with both risk and effort choices is more serious than either a pure risk choice problem or a pure effort choice problem. For either problem by itself, the assumption of risk neutrality implies that, despite the limitations on the entrepreneur's ability to pay, there always exists an incentive scheme which implements a first-best outcome. For the integrated problem studied here, such an incentive scheme never exists if the entrepreneur's initial wealth is insufficient to finance the first-best level of investment.

If the entrepreneur's initial wealth is insufficient to finance the firstbest level of investment, second-best outcomes always involve excessive risk taking in the sense that a marginal increase in effort designed to reduce the entrepreneur's failure risk would raise the expected aggregate surplus. If the technology is separable, failure risk is strictly greater in a second-best outcome than in a first-best outcome.

By contrast, the characterization of second-best levels of investment and effort depends on the underlying technology, in particular, the elasticity of substitution between these two inputs. If the elasticity of substitution between investment and effort is equal to one, second-best investment and effort levels are both below their surplus-maximizing values, but, conditional on second-best risk and effort choices, the investment level is actually efficient. If the elasticity of substitution between investment and effort differs from one, the difference affects the wedges in the conditions for both, effort and investment, enhancing one wedge and reducing the other, so that, conditional on second-best risk and effort choices, there is overinvestment if the elasticity of substitution is less than one and underinvestment if the

\footnotetext{
${ }^{4}$ The exception is Holmström and Milgrom (1987). However, in their model, moral hazard concerns effort choice only, and they are quite explicit that the model cannot be extended to allow for risk choices as well (1987, p. 324).
} 
elasticity of substitution is greater than one. The overall size of the venture is always too small in that a proportional increase in both effort and investment would raise expected aggregate surplus.

If investment and effort are close to being perfect substitutes, a secondbest contract comes close to maximizing the expected aggregate surplus. In this case, the level of outside finance is close to zero, and the entrepreneur's effort substitutes for the shortfall in investment. If investment and effort are close to being perfect complements, a second-best contract also comes close to maximizing the expected aggregate surplus. In this case, investment, effort, and risk choices are close to their first-best values; the investmenteffort ratio is slightly above the first-best level.

These findings suggest that, in assessing the impact of double moral hazard, we must pay more attention to the technology. Relations of complementarity and substitutability affect the severity of the agency problem, as well as the comparative statics properties of the different decision variables.

As for the research program of "explaining" capital structure in terms of the incentive implications of the return pattern of the entrepreneur's retained inside equity, the paper's results are ambivalent: On the one hand, it provides an interesting class of cases in which optimal incentive schemes can indeed take a form that corresponds to a combination of debt and equity issues. On the other hand, it also gives examples where optimal incentive schemes cannot be interpreted in terms of debt and equity packages. Even in those cases where optimal incentive schemes can be generated by debt and equity instruments, this finding seems to reflect a certain arbitrariness of optimal schemes under risk neutrality, rather than any inherent structural properties of these financial instruments.

In the following, Section 2 introduces the basic model, presents the assumptions about the technology, and demonstrates the impossibility of implementing first-best when the entrepreneur has insufficient funds of his own. Section 3 provides a systematic analysis of second-best contracts under the assumption that the observation of return realizations involves no "noise" and permits a precise inference about certain aspects of the entrepreneur's choices. This section contains the results on excessive risk taking and on the role of the elasticity of substitution between investment and effort that were mentioned above. Section 4 gives conditions under which the second-best outcomes considered in Section 3 are robust to the introduction of "noise". Section 5 discusses whether the incentive schemes that are used to support these outcomes can be interpreted as the results of outside debt and equity finance. 


\section{A Model of Outside Finance with Double Moral Hazard}

\subsection{The Model}

An entrepreneur with initial assets $A \geq 0$ wants to raise external funds $I-A$ in order to finance a project with an overall investment $I$. When the investment is made, he chooses an effort level $\ell$ from a set $\mathcal{L} \subset \mathbb{R}_{+}$and a risk class $\pi$ from a set $\Pi \subset \mathbb{R}_{+}$. Given $I, \ell, \pi$, the project earns the random gross return $\tilde{y}$ where

$$
\tilde{y}=\tilde{\theta} f(I, \ell, \pi) \quad \text { with probability } \pi
$$

and

$$
\tilde{y}=0 \quad \text { with probability } 1-\pi .
$$

In $(2.1), \tilde{\theta}$ is a positive-valued random variable with a given distribution function $F$; without loss of generality, I assume that $E \tilde{\theta}=1$. The risk class $\pi$ is identified with the project's success probability. A project of risk class $\pi$ fails altogether with probability $1-\pi$. The project's return in the event of success depends on the effort $\ell$ and the risk class $\pi$, as well as the investment $I$. The function $f$ is increasing in $I$ and $\ell$ and decreasing in $\pi$. An increase in the success probability $\pi$ necessitates either a decrease in the project's return in the event of success or an increase in $I$ or $\ell$.

The functions $F$ and $f$ are assumed to be common knowledge. I also assume that the investment $I$ is observable and verifiable. However, outside financiers cannot observe the chosen effort level $\ell$ and risk class $\pi$. These choices will depend on the incentives that the finance contract provides to the entrepreneur.

Besides the investment $I$ and the outside funding $I-A$, the contract must stipulate the division of the gross return $\tilde{y}$ between the entrepreneur and his financiers. I assume that the realizations of $\tilde{y}$ are observable and verifiable. The portion $\tilde{w}$ of the return that goes to the entrepreneur and the portion $\tilde{r}=\tilde{y}-\tilde{w}$ that goes to the financiers can therefore be taken to be arbitrary functions of $\tilde{y}$, without any additional incentive considerations. ${ }^{5}$

The entrepreneur and the financiers are taken to be risk neutral. Expected payoffs are specified as $E \tilde{w}-\ell$ for the entrepreneur and $E \tilde{r}-(I-A)$ for the financiers. Because of risk neutrality, there is no loss of generality in assuming that $\tilde{w}$ and $\tilde{r}=\tilde{y}-\tilde{w}$ are given by deterministic functions of $\tilde{y}$;

\footnotetext{
${ }^{5}$ The setting is thus different from the costly-state-verification literature initiated by Townsend (1979) and Gale and Hellwig (1985).
} 
any randomization in $\tilde{w}$ conditional on $\tilde{y}$ could be averaged out without any change in expected payoffs and in incentives. Expected payoffs can therefore be written as

$$
U(I, \ell, \pi, w(\cdot))=\pi \int w(\theta f(I, \ell, \pi)) d F(\theta)+(1-\pi) w(0)-\ell
$$

for the entrepreneur and

$$
V(I, \ell, \pi, w(\cdot))=\pi \int[\theta f(I, \ell, \pi)-w(\theta f(I, \ell, \pi))] d F(\theta)-(1-\pi) w(0)-(I-A)
$$

for the financiers.

If there is Bertrand competition among financiers, the overall contracting problem can be written as

$$
\max _{\substack{I \geq 0, \ell \in \mathcal{L} \\ \pi \in \Pi, w(\cdot) \in \mathcal{W}}} U(I, \ell, \pi, w(\cdot))
$$

subject to the financiers' participation constraint

$$
V(I, \ell, \pi, w(\cdot)) \geq 0
$$

and the incentive compatibility constraint

$$
U(I, \ell, \pi, w(\cdot)) \geq U\left(I, \ell^{\prime}, \pi^{\prime}, w(\cdot)\right) \quad \text { for all } \ell^{\prime} \in \mathcal{L} \text { and all } \pi^{\prime} \in \Pi .
$$

In this formulation, $\mathcal{W}$ is a set of admissible incentive schemes.

Problem (2.5) encompasses several specifications of agency problems in the literature as special cases:

- Jensen and Meckling's (1976) analysis of effort choice and equity finance corresponds to the specification

$$
\tilde{\theta} \equiv 1, \mathcal{L}=\mathbb{R}_{+}, \Pi=\{1\},
$$

and

$$
\mathcal{W}=\{w(\cdot) \mid \text { for some } \alpha \in[0,1], w(y)=(1-\alpha) y \text { for all } y\} .
$$

In this specification, $\alpha$ is the share of outside equity in the firm. 
- The analysis of risk choice and debt finance in Jensen and Meckling (1976), Keeton (1979), or Stiglitz and Weiss (1981) corresponds to the specification

$$
\mathcal{L}=\{0\}, \Pi=[0,1]
$$

and

$$
\mathcal{W}=\{w(\cdot) \mid \text { for some } R \geq 0, w(y)=\max (0, y-R) \text { for all } y\} .
$$

In this specification, $R$ is the entrepreneur's debt service obligation to his creditors.

- The analysis of effort choice under any form of outside finance in Innes (1990) corresponds to the case where $\tilde{\theta}$ is a nondegenerate random variable with range $\mathbb{R}_{+}, \mathcal{L}=\mathbb{R}_{+}, \Pi=\{1\}$, and

$$
\begin{aligned}
\mathcal{W}= & \{w(\cdot) \mid w(y) \geq 0, y-w(y) \geq 0 \text { for all } y \\
& \text { and } y-w(y) \text { is nondecreasing in } y .
\end{aligned}
$$

By contrast to these contributions, I will consider the general specification

$$
\mathcal{L}=\mathbb{R}_{+}, \Pi=[0,1]
$$

and

$$
\mathcal{W}=\{w(\cdot) \mid w(y) \geq 0 \text { für all } y\} .
$$

Thus, I allow for moral hazard with respect to effort and risk choices at the same time. Moreover, I impose hardly any restrictions on the set of admissible incentive schemes. I propose this generalization for the following reasons.

- First, if the research program is to explain observed financing patterns in terms of optimal incentive contracting, one does not want to impose the prior assumption that the implied incentive scheme must take the form $w(y)=(1-\alpha) y$ or even $w(y)=(1-\alpha) \max (0, y-R)$. If such an assumption were imposed, one could not really claim to explain the use of contracts that correspond to a mix of debt and equity issues. ${ }^{6}$ Therefore, I only impose the condition that $w(y)$ be nonnegative, so that $r(y)=y-w(y)$, the portion of the return that goes to the financiers, does not exceed $y$. This condition is motivated

\footnotetext{
${ }^{6}$ This point underlies the difference between Townsend (1979), Gale and Hellwig (1985), and Innes (1990) on the one hand and Jensen and Meckling (1976) on the other hand.
} 
by the consideration that any source of funds that is available to the entrepreneur is already included in the initial assets $A$. Once these assets are brought into the firm, there are no further funds to provide for payments in excess of $y$ going to the financiers.

- Second, if one believes that the mix of outside debt and equity financing of a firm represents an optimal response to a combination of moral-hazard problems involving risk choices and effort choices, then one ought to study the matter in a model that actually involves a double moral-hazard problem. Separate analyses of moral hazard with respect to effort choices and of moral hazard with respect to risk choices are not enough because such analyses cannot take account of the interdependence between the two moral-hazard problems.

\subsection{A Reformulation of the Contracting Problem}

To highlight the interdependence of effort choices and risk choices, I introduce the variable

$$
\bar{y}=f(I, \ell, \pi) .
$$

This variable represents the conditional expectation of the random return $\tilde{y}$ given the event of success. According to equation (2.1), the impact of $\ell$ and $\pi$ on the conditional distribution of $\tilde{y}$ given the event of success is entirely determined by their impact on $\bar{y}$. In particular, the conditional expectation of the incentive payment $\tilde{w}=w(\tilde{y})$ given the event of success is equal to $\bar{w}(\bar{y}):=\int w(\theta \bar{y}) d F(\theta)$, which depends on $\ell$ and $\pi$ only through the effects of $\ell$ and $\pi$ on $\bar{y}$. The overall incentive problem can therefore be decomposed into two subproblems:

- Taking $\bar{y}$ as given, how can the entrepreneur be motivated to choose the desired combination of $\ell$ and $\pi$ to achieve $\bar{y}$ ?

- How can the entrepreneur be motivated to aim for the desired $\bar{y}$ ?

In the first of these subproblems, one has to take account of the fact that a given $\bar{y}$ can be achieved by a low-effort/high-risk strategy, as well as a high-effort/low-risk strategy. Financiers would prefer the latter, but the entrepreneur is unwilling to comply unless he is given proper incentives. This requires that the difference $\bar{w}(\bar{y})-w(0)$ between the conditional expectations of payments in the event of success and in the event of failure must be sufficiently large. 
Proceeding formally, let

$$
\mathcal{D}:=\left\{(\bar{y}, I, \pi) \in \mathbb{R}_{+}^{2} \times[0,1] \mid f(I, \ell, \pi) \geq \bar{y} \text { for some } \ell\right\},
$$

and, for $(\bar{y}, I, \pi) \in \mathcal{D}$, let

$$
\lambda(\bar{y}, I, \pi)=\min \{\ell \geq 0 \mid f(I, \ell, \pi) \geq \bar{y}\}
$$

be the smallest effort level that achieves the conditionally expected return $\bar{y}$ in the event of success. Further, for any $\bar{y}, v$, and $I$, define

$$
U^{*}(\bar{y}, v, I):=\sup _{\pi \in[0,1]}[\pi v-\lambda(\bar{y}, I, \pi)] .
$$

Problem (2.5), with the constraints (2.6) and (2.7), can then be rewritten as:

$$
\max _{\substack{(\bar{y}, I, \pi) \in \mathcal{D} \\ w(\cdot) \in \mathcal{W}}}[\pi \bar{w}(\bar{y})+(1-\pi) w(0)-\lambda(\bar{y}, I, \pi)]
$$

subject to the constraints that

$$
\begin{gathered}
\pi(\bar{y}-\bar{w}(\bar{y}))-(1-\pi) w(0)-(I-A) \geq 0, \\
\pi(\bar{w}(\bar{y})-w(0))-\lambda(\bar{y}, I, \pi)=U^{*}(\bar{y}, \bar{w}(\bar{y})-w(0), I),
\end{gathered}
$$

and

$$
U^{*}(\bar{y}, \bar{w}(\bar{y})-w(0), I) \geq U^{*}\left(\bar{y}^{\prime}, \bar{w}\left(\bar{y}^{\prime}\right)-w(0), I\right)
$$

for all $\bar{y}^{\prime}$ such that $\left(\bar{y}^{\prime}, I, \pi\right) \in \mathcal{D}$; in $(2.19)-(2.22), \bar{y}=f(I, \ell, \pi), U^{*}(\cdot, \cdot, \cdot)$ is defined by (2.18) and, for any $\bar{y}^{\prime}$,

$$
\bar{w}\left(\bar{y}^{\prime}\right):=\int w\left(\theta \bar{y}^{\prime}\right) d F(\theta) .
$$

A solution to this problem will be referred to as an optimal contract.

In this formulation, the incentive constraint (2.7) has been decomposed into two conditions: For the stipulated $\bar{y}, I$, and $v=\bar{w}(\bar{y})-w(0)$, the desired $\pi$ must achieve the supremum in (2.18), and the entrepreneur must consider $\bar{y}$ to be at least as attractive as any other return target $\bar{y}^{\prime}$.

The new problem formulation makes the formal structure of the overall incentive problem clearer. Except for incentive considerations, the financiers do not care about the entrepreneur's effort at all. They care about the conditional expectation $\bar{y}$ of returns given the event of success, the investment level $I$, and the success probability $\pi$. From their perspective, the problem 
is to motivate the entrepreneur to put in the effort $\lambda(\bar{y}, I, \pi)$ that will, for given $I$, produce the contractually stipulated pair $(\bar{y}, \pi)$. In this problem, the variables $\bar{y}$ and $\pi$ play very different roles. Neither variable is directly observable. However, some information about $\bar{y}$ is provided by the return $\tilde{y}=\tilde{\theta} \bar{y}$ in the event of success; when there is no noise, i.e., in the case $\tilde{\theta} \equiv 1$, $\bar{y}$ is actually revealed by $\tilde{y}$. By contrast, the only observable variable that provides information about the success probability $\pi$ is the incidence of success or failure as such. To meet the incentive constraint (2.21), therefore, the incentive payment to the entrepreneur in the event of success must involve a sufficiently high premium over the payment he receives in the event of failure.

\subsection{Basic Assumptions and Preliminary Lemmas}

Throughout the analysis, I assume that the function $f$ is continuous on $\mathbb{R}_{+}^{2} \times[0,1]$, as well as twice continuously differentiable on $\mathbb{R}_{++}^{2} \times(0,1)$. Moreover,

$$
f(I, \ell, 1)=f(0,0, \pi)=0
$$

for all $I, \ell, \pi$. On $\mathbb{R}_{++}^{2} \times(0,1), f$ is strictly increasing in $I$ and $\ell$ as well as decreasing in $\pi$, i.e.,

$$
f_{I}(I, \ell, \pi)>0, f_{\ell}(I, \ell, \pi)>0, f_{\pi}(I, \ell, \pi)<0 .
$$

Further, $f$ is assumed to be differentiably strictly concave in $I$ and $\ell$, concave in $\pi$, and differentiably strictly quasiconcave in $\ell$ and $\pi .^{7}$

Finally, to eliminate the possibility of boundary solutions, I impose the boundary conditions that

$$
\lim _{I^{\prime} \downarrow 0} f_{I}\left(I^{\prime}, \ell, \pi\right)=\infty, \lim _{\ell^{\prime} \downarrow 0} f_{\ell}\left(I, \ell^{\prime}, \pi\right)=\infty
$$

for all $I>0, \ell>0$, and $\pi \in(0,1)$, that, for some $\lambda_{0}$ and $\pi_{0}$, one has

$$
\lim _{I \downarrow 0} \frac{f\left(I, \lambda_{0} I, \pi_{0}\right)}{I}=\infty
$$

and that, for any sequence $\left\{\left(I^{k}, \ell^{k}, \pi^{k}\right)\right\}$ with $I^{k}+\ell^{k}$ going out of bounds, one has

$$
\lim _{k \rightarrow \infty} \frac{f\left(I^{k}, \ell^{k}, \pi^{k}\right)}{I^{k}+\ell^{k}}=0 .
$$

\footnotetext{
${ }^{7}$ The qualifier "differentiably" means that the corresponding measures of curvature in terms of second derivatives are nonzero. Thus, the Hessian of $f$ with respect to $I$ and $\ell$ is assumed to be negative definite, and the $\pi-\ell$ - isoquants of $f$ are assumed to have strictly positive Gaussian curvature.
} 
The following lemma uses these boundary conditions to show that, under an optimal contract, the variables $\bar{y}, I, \ell$, and $\pi$ do not take boundary values. ${ }^{8}$ The proof is given in the Appendix.

Lemma 2.1 Any optimal contract satisfies $\bar{y}>0, I>0, \ell>0, \pi \in(0,1)$, and

$$
U^{*}(\bar{y}, \bar{w}(\bar{y})-w(0), I)=\pi \bar{y}-\ell-I+A>A .
$$

The following lemma provides a characterization of the effort cost function $\lambda$ that was defined in (2.17) and (2.16) above.

Lemma 2.2 The effort cost function $\lambda$ is continuous on its domain $\mathcal{D}$. At any point $(\bar{y}, I, \pi) \in \mathcal{D}$ where $\lambda(\bar{y}, I, \pi)>0$, the effort cost function is twice continuously differentiable, strictly increasing in $\bar{y}$, strictly decreasing in $I$, and strictly increasing in $\pi$. The effort cost function is also differentiably strictly convex in $\bar{y}$ and $I$ and differentiably strictly convex in $\pi$.

Proof. Continuity of $\lambda$ follows from the maximum theorem. Differentiability at any point $(\bar{y}, I, \pi)$ at which $\lambda(\bar{y}, I, \pi)>0$ follows from the implicit function theorem. The derivatives are given as

$$
\begin{gathered}
\lambda_{\bar{y}}(\bar{y}, I, \pi)=\frac{1}{f_{\ell}(I, \lambda(\bar{y}, I, \pi), \pi)}>0, \\
\lambda_{I}(\bar{y}, I, \pi)=-\frac{f_{I}(I, \lambda(\bar{y}, I, \pi), \pi)}{f_{\ell}(I, \lambda(\bar{y}, I, \pi), \pi)}<0,
\end{gathered}
$$

and

$$
\lambda_{\pi}(\bar{y}, I, \pi)=-\frac{f_{\pi}(I, \lambda(\bar{y}, I, \pi), \pi)}{f_{\ell}(I, \lambda(\bar{y}, I, \pi), \pi)}>0 .
$$

Differentiably strict convexity in $\bar{y}$ and $I$, as well as differentiably strict convexity in $\pi$ follow by routine calculations, e.g.,

$$
\begin{aligned}
\lambda_{\pi \pi}(\bar{y}, I, \pi) & =-\frac{f_{\ell} f_{\pi \pi}+f_{\ell} f_{\pi \ell} \lambda_{\pi}-f_{\pi} f_{\ell \pi}-f_{\pi} f_{\ell \ell} \lambda_{\pi}}{f_{\ell}^{2}} \\
& =-\frac{f_{\ell}^{2} f_{\pi \pi}-2 f_{\pi} f_{\ell} f_{\ell \pi}+f_{\pi}^{2} f_{\ell \ell}}{f_{\ell}^{3}}
\end{aligned}
$$

\footnotetext{
${ }^{8}$ The reader should note that the lemma requires the "joint" Inada condition $(2.27)$ as well as the separate conditions in $(2.26)$. For instance, the specification $f(I, \ell, \pi)=$ $X(\pi) c \ln \left(1+(I \ell)^{\frac{1}{2}}\right)$ satisfies $(2.26)$, but, if $c \pi X(\pi) \leq \sqrt{8}$ for all $\pi$, one finds that, for $A=0$, there is no contract with $I>0$ that satisfies the financiers' participation constraint as well as the entrepreneur's incentive constraints. Yet, for this specification, first-best investment and effort levels are strictly positive if $c \pi X(\pi)>2$ for some $\pi$.
} 
which is positive by the assumption that $f$ is differentiably strictly quasiconcave in $\ell$ and $\pi$.

Strict convexity of $\lambda$ in $\pi$ implies that the entrepreneur's objective function in (2.18) is strictly concave. The incentive constraint (2.21) can therefore be handled by a first-order approach. Formally, $\lambda_{\pi \pi}>0$ implies:

Lemma 2.3 A contract $(I, \ell, \pi, \bar{y}, w(\cdot))$ with $(\bar{y}, I, \pi) \in \mathbb{R}_{++}^{2} \times(0,1)$ satisfies the incentive constraint (2.21) if and only if it satisfies the first-order condition

$$
\bar{w}(\bar{y})-w(0)=\lambda_{\pi}(\bar{y}, I, \pi)
$$

Because financiers have no direct control over the entrepreneur's choice of $\pi$, the only way to implement the stipulated $\pi$ is to provide appropriate monetary incentives through the premium $\bar{w}(\bar{y})-w(0)$ of the entrepreneur's expected return in the event of success over his return in the event of failure.

\subsection{The Impossibility of Implementing First-Best}

The need for such monetary incentives may collide with the financiers' desire to recover the opportunity cost of their funds. For a given triple $(\bar{y}, I, \pi)$, it may not be possible to find a contract that implements $(\bar{y}, I, \pi)$ and satisfies the financiers' participation constraint. As an example, consider an outcome $\left(\bar{y}^{*}, I^{*}, \pi^{*}\right)$ that maximizes the expected aggregate surplus $\pi \bar{y}-I-\lambda(\bar{y}, I, \pi) .{ }^{9}$ Such a first-best outcome satisfies the first-order condition

$$
\bar{y}^{*}=\lambda_{\pi}\left(\bar{y}^{*}, I^{*}, \pi^{*}\right) .
$$

Implementation of $\left(\bar{y}^{*}, I^{*}, \pi^{*}\right)$ through a contract satisfying the incentive constraint $(2.21)$ would therefore require that $\bar{w}\left(\bar{y}^{*}\right)-w(0)=\bar{y}^{*}$. The premium of the entrepreneur's return in the event of success over his return in the event of failure would then be equal to the entire return from the success of his venture. If the entrepreneur's own funds do not suffice to finance the first-best investment $I^{*}$, such an arrangement violates the financiers' participation constraint. Thus, one obtains:

Proposition 2.4 A first-best outcome with $I^{*}>A$ cannot be implemented.

\footnotetext{
${ }^{9}$ Because I have not assumed that the product $\pi f(I, \ell, \pi)$ is concave, there may be more than one such triple.
} 
The logic behind this result is the same as the logic behind the results of Jensen and Meckling (1976) that, if the entrepreneur has not enough funds of his own, then, in a model of moral hazard with respect to effort, a first-best outcome cannot be reached by issuing equity to outsiders, and, in a model of moral hazard with respect to risk-taking, a first-best outcome cannot be reached by issuing debt to outsiders. ${ }^{10}$ By contrast to these results, Proposition 2.4 makes no assumption about the form of the finance contract. In the pure effort choice model of Jensen and Meckling, a firstbest outcome can be implemented by using debt finance rather than equity finance. In the pure risk choice model of Jensen and Meckling, Keeton, or Stiglitz and Weiss, a first-best outcome can be implemented by using equity rather than debt finance. ${ }^{11}$ In the combined effort-and-risk choice problem studied here, the implementability of a first-best outcome cannot be reestablished by changing the class of incentive schemes that are considered. The combined effort-and-risk choice problem is thus more robust than either the pure effort choice problem or the pure risk choice problem alone.

\section{Optimal Contracts in the Absence of Noise}

\subsection{Optimality Conditions for the Case $\tilde{\theta} \equiv 1$}

Lemma 2.3 implies that the incentive constraint (2.21) can be replaced by equation (2.33). This equation can also be used to substitute for $\bar{w}(\bar{y})$ in the objective function (2.19) and the financiers' participation constraint (2.20). The contracting problem then takes the form

$$
\max _{\substack{I \geq 0, \pi \in[0,1] \\ \bar{y} \geq 0, w(\cdot)}}\left[\pi \lambda_{\pi}(\bar{y}, I, \pi)+w(0)-\lambda(\bar{y}, I, \pi)\right]
$$

subject to the incentive constraints (2.33), (2.22), and (2.23) and the participation constraint

$$
\pi\left(\bar{y}-\lambda_{\pi}(\bar{y}, I, \pi)\right)-w(0)-(I-A) \geq 0 .
$$

In this section, I study this problem under the additional assumption that $\tilde{\theta} \equiv 1$. Under this assumption, the observed return $\tilde{y}$ in the event of

\footnotetext{
${ }^{10}$ On the latter result, see also Stiglitz and Weiss (1981) and Bester and Hellwig (1987).

${ }^{11}$ As discussed by Bester and Hellwig (1987), the analysis of moral hazard with respect to risk choices in Stiglitz and Weiss (1981) presumes that financiers cannot observe the level of returns in the event of success; otherwise, equity finance would solve the problem.
} 
success is equal to the target return $\bar{y}$, so $(2.23)$ implies $\bar{w}(y)=w(y)$ for all $y$. By observing the entrepreneur's return in the event of success, the financiers obtain full information about the target that the entrepreneur was aiming for. Therefore, a forcing device can be used to ensure that the incentive compatibility condition (2.22) is satisfied.

Lemma 3.1 If $\tilde{\theta} \equiv 1$, any contract $(I, \ell, \pi, \bar{y}, w(\cdot))$ that satisfies

$$
\begin{gathered}
w(y)=w(0) \quad \text { if } y<\bar{y}, \\
w(y)=w(0)+\lambda_{\pi}(\bar{y}, I, \pi) \quad \text { if } y \geq \bar{y},
\end{gathered}
$$

and

$$
U^{*}(\bar{y}, w(\bar{y})-w(0), I) \geq 0,
$$

is incentive-compatible.

Proof. If $\tilde{\theta} \equiv 1$, then, for any contract satisfying (2.23), equations (3.3) and (3.4) imply

$$
\bar{w}(y)=w(0) \text { if } y<\bar{y}
$$

and

$$
\bar{w}(y)=w(0)+\lambda_{\pi}(\bar{y}, I, \pi) \text { if } y \geq \bar{y} .
$$

By Lemma 2.3, (3.7) implies that the incentive constraint (2.21) is satisfied.

I next consider the incentive constraint (2.22). For $\bar{y}^{\prime}<\bar{y},(3.6)$ and the specification of $U^{*}$ imply

$$
U^{*}\left(\bar{y}^{\prime}, \bar{w}\left(\bar{y}^{\prime}\right)-w(0), I\right)=-\lambda\left(\bar{y}^{\prime}, I, 0\right) \leq 0 .
$$

The inequality (2.22) follows from (3.8) and (3.5). For $\bar{y}^{\prime}>\bar{y},(3.7)$ and (3.4) imply that

$$
U^{*}\left(\bar{y}^{\prime}, \bar{w}\left(\bar{y}^{\prime}\right)-w(0), I\right)=U^{*}\left(\bar{y}^{\prime}, w(\bar{y})-w(0), I\right) .
$$

Because, by inspection of (2.18) and Lemma 2.2, $U^{*}$ is nonincreasing in output, the inequality (2.22) holds in this case as well.

Lemma 3.1 implies that, when $\tilde{\theta} \equiv 1$, the constraints $(2.33),(2.22)$, and (2.23) are moot in the sense that, for given $\bar{y}, I, \pi$, and $w(0)$, they are automatically satisfied if $\bar{w}(\cdot)=w(\cdot)$ and $w(\cdot)$ satisfies (3.3) and (3.4). Because the values of $\bar{w}(\cdot)$ and $w(\cdot)$ at $y \neq 0$ do not enter the entrepreneur's objective function in (3.1) or the financiers' participation constraint (3.2), the 
contracting problem simplifies to the problem of choosing scalars $\bar{y}, I$, $\pi$, and $w(0)$ so as to maximize the objective in (3.1) subject to (3.2).

The Lagrangian for this problem can be written as:

$$
\pi \bar{y}-\lambda(\bar{y}, I, \pi)-I+A+(\mu-1)\left[\pi\left(\bar{y}-\lambda_{\pi}(\bar{y}, I, \pi)\right)-w(0)-(I-A)\right] .
$$

The necessary first-order conditions can be written as:

$$
\begin{gathered}
\pi-\lambda_{\bar{y}}=\frac{\mu-1}{\mu}\left(\pi \lambda_{\pi \bar{y}}-\lambda_{\bar{y}}\right), \\
-\lambda_{I}-1=\frac{\mu-1}{\mu}\left(\pi \lambda_{\pi I}-\lambda_{I}\right), \\
\bar{y}-\lambda_{\pi}=\frac{\mu-1}{\mu} \pi \lambda_{\pi \pi},
\end{gathered}
$$

and

$$
\mu-1 \geq 0,
$$

with equality if $w(0)>0$; in $(3.11)-(3.13)$, the terms $\lambda_{\bar{y}}, \lambda_{\pi \bar{y}}$, etc. are all evaluated at $(\bar{y}, I, \pi)$. The left-hand sides of equations (3.11) - (3.13) indicate the impact of the maximization variables on the aggregate surplus $\pi \bar{y}-\lambda-I$. The right-hand sides reflect the differences between the impact of maximization variables on the financiers' participation constraint and on the aggregate surplus. These differences are relevant because, from the financiers' point of view, the effort cost of the outcome $(\bar{y}, I, \pi)$ corresponds to the expected incentive payment $\pi \lambda_{\pi}(\bar{y}, I, \pi)$, rather than the actual cost $\lambda(\bar{y}, I, \pi)$.

\subsection{Excessive Risk Taking}

If $\mu=1$, equations (3.11) - (3.13) reduce to the first-order conditions maximizing the aggregate surplus $\pi \bar{y}-\lambda-I$. By the argument used to esatblish Proposition 2.4, this is only possible if $A \geq I$. If $A<I$, one must have $\mu>1$ and $w(0)=0$.

If $\mu>1$, equation (3.13) implies that, at an optimal contract, the success probability $\pi$ is fixed at a value that is inefficiently low in the sense that a small increase in $\pi$ would raise expected returns by more than the entrepreneur's effort cost. The reason is that, with $\lambda_{\pi \pi}>0$, an increase in $\pi$ necessarily raises the expected incentive payment $\pi \lambda_{\pi}$ by more than the actual effort cost $\lambda$. Given the convexity of the effort cost function in $\pi$, this observation yields: 
Proposition 3.2 Assuming that $\tilde{\theta} \equiv 1$, let $(I, \ell, \pi, \bar{y}, w(\cdot))$ be an optimal contract, and let $\hat{\pi}=\arg \max _{\rho}[\rho \bar{y}-\lambda(\bar{y}, I, \rho)-I]$. If $A<I$, then

$$
\pi<\hat{\pi} \text { and } \ell<\lambda(\bar{y}, I, \hat{\pi})
$$

Conditional on the other variables of the contract, an optimal contract always involves excessive risk taking and too little effort to reduce the probability of failure. The reason is that the tradeoff between the success probability $\pi$ and the effort $\lambda(\bar{y}, I, \pi)$ that is relevant for aggregate surplus is more favourable than the tradeoff between the success probability $\pi$ and the requisite incentive payment $\pi \lambda_{\pi}(\bar{y}, I, \pi)$. To the extent that the participation constraint $\pi \bar{y}-\pi \lambda_{\pi}(\bar{y}, I, \pi) \geq I-A$ for the financiers is relevant, the latter tradeoff plays a role and contributes to reducing both $\pi$ and $\lambda(\bar{y}, I, \pi)$. The result can be understood as an instance of the general principle that, in the presence of agency costs, some deviation from first-best is desirable because, at a first-best outcome, the efficiency loss of such a deviation is small relative to the savings in agency costs. ${ }^{12}$

All this is conditional on the other variables of the optimal contract. To move beyond such a conditional assessment, I impose the additional assumption that the return function $f$ takes separable form

$$
f(I, \ell, \pi)=X(\pi) g(I, \ell)
$$

For this specification, I will show that, if the entrepreneur needs outside funds, then the success probability $\pi$ under an optimal contract is smaller than the success probability in a first-best outcome.

I being with two lemmas showing that, for the separable specification (3.16), the first-best outcome is unique and that one must have $\mu>1$ if the entrepreneur's own funds are less than the first-best investment level.

Lemma 3.3 If $f$ takes the separable form (3.16), there is a unique first-best outcome $\left(\bar{y}^{*}, I^{*}, \ell^{*}, \pi^{*}\right)$. The first-best success probability $\pi^{*}$ is the unique solution to the equation

$$
X\left(\pi^{*}\right)+\pi^{*} X^{\prime}\left(\pi^{*}\right)=0 .
$$

\footnotetext{
${ }^{12}$ This principle seems to have been first established in the Ramsey-Boiteux theory of optimal indirect taxation and public-sector pricing, and, indeed, equations (3.11) - (3.13) bear some semblance to optimal tax formulae. For early statements in the context of agency models with hidden actions, see Holmström (1979) and Shavell (1979).
} 
Proof. For the separable specification (3.16), any first-best outcome involves a risk choice $\pi^{*}$ that maximizes $\left[\pi X(\pi) g\left(I^{*}, \ell^{*}\right)-I^{*}-\ell^{*}\right]$ and therefore also $\pi X(\pi)$. Under the given assumptions on $f$, the function $X(\cdot)$ in the separable specification (3.16) is decreasing and concave. The second derivative of the product $\pi X(\pi)$ with respect to $\pi, \pi X^{\prime \prime}+2 X^{\prime}$, is therefore negative, and the product $\pi X(\pi)$ is strictly concave in $\pi$. The solution to the problem $\max \pi X(\pi)$ is unique and coincides with the unique solution to equation (3.17). A first-best outcome also satisfies $\left(\bar{y}^{*}, I^{*}\right) \in$ $\arg \max \left[\pi^{*} \bar{y}-\lambda\left(\bar{y}, I, \pi^{*}\right)-I+A\right]$. By Lemma 2.2 , the solution to this problem is unique.

Lemma 3.4 If $f$ takes the separable form (3.16) and if $A<I^{*}$, then, in the first-order conditions for an optimal contract, one has $\mu>1$ and $w(0)=0$.

Proof. If $f$ takes the form (3.16), (2.30) - (2.32) take the form

$$
\lambda_{\bar{y}}=\frac{1}{X g_{\ell}}, \lambda_{I}=-\frac{g_{I}}{g_{\ell}}, \lambda_{\pi}=-\frac{X^{\prime}}{X} \frac{g}{g_{\ell}} .
$$

Therefore, if $\mu=1$, conditions (3.11) - (3.13) imply $\pi X(\pi) g_{\ell}=1, g_{I}=g_{\ell}$, and $X(\pi) g=-\frac{X^{\prime}(\pi) g}{X(\pi) g_{\ell}}$. The first and last of these conditions together yield $X=-\pi X^{\prime}(\pi)$. Then $\pi$ must be a solution to equation (3.17). By Lemma 3.3 , this implies $\pi=\pi^{*}$. Given that $\pi=\pi^{*}$, the strict concavity of $g$ implies that the conditions $\pi X(\pi) g_{\ell}=1$ and $g_{I}=g_{\ell}$ have a unique solution; moreover, for $\pi=\pi^{*}$, this solution must coincide with the first-best input pair $\left(I^{*}, \ell^{*}\right)$. For $f$ taking the form $(3.16), \mu=1$ thus implies that the outcome $(\bar{y}, I, \ell, \pi)$ is equal to the unique first-best outcome $\left(\bar{y}^{*}, I^{*}, \ell^{*}, \pi^{*}\right)$. By Proposition 2.4, it follows that $A \geq I^{*}$. Conversely, if $A<I^{*}$, one cannot have $\mu=1$.

Given these lemmas, the following result translates the conditional assessment of the second-best choice of $\pi$ that is provided by Proposition 3.2 into a comparison of $\pi$ with the first-best success probability $\pi^{*}$.

Proposition 3.5 Assume that $\tilde{\theta} \equiv 1$. If $f$ takes the separable form (3.16) and if $A<I^{*}$, then, any optimal contract satisfies $\pi<\pi^{*}$.

Proof. From (3.18), one computes

$$
\lambda_{\pi \pi}=\frac{-X X^{\prime \prime}+X^{\prime 2}}{X^{2}} \frac{g}{g_{\ell}}+\lambda_{\pi \bar{y}} \frac{\lambda_{\pi}}{\lambda_{\bar{y}}} .
$$


Condition (3.13) therefore takes the form:

$$
X g-\lambda_{\pi}=\frac{\mu-1}{\mu} \pi\left(\frac{-X X^{\prime \prime}+X^{\prime 2}}{X^{2}} \frac{g}{g_{\ell}}+\lambda_{\pi \bar{y}} \frac{\lambda_{\pi}}{\lambda_{\bar{y}}}\right) .
$$

If one multiplies (3.11) by $\lambda_{\pi} / \lambda_{\bar{y}}$ and subtracts the resulting equation from (3.20), one obtains

$$
\left(X+\pi X^{\prime}\right) g=\frac{\mu-1}{\mu}\left(\pi \frac{\left(-X X^{\prime \prime}+X^{\prime 2}\right)}{X^{2}}+\frac{\left(-X^{\prime}\right)}{X}\right) \frac{g}{g_{\ell}} .
$$

Because $\mu>1$ and because, under the given assumptions on $f$, the function $X(\cdot)$ is decreasing and concave, the right-hand side of (3.21) is positive. Therefore, one has

$$
X(\pi)+\pi X^{\prime}(\pi)>0 .
$$

Given the concavity of the function $\pi \rightarrow \pi X(\pi)$ and the first-order condition (3.17) for $\pi^{*},(3.22)$ yields $\pi<\pi^{*}$.

\subsection{Investment and Effort Levels: The Cobb-Douglas Case}

The conditions for $\bar{y}$ and $I$, equations (3.11) and (3.12), are more difficult to

disentangle. They do not involve any incentive considerations concerning the variables $\bar{y}$ and $I$ as such. They reflect the consideration that $\bar{y}$ and $I$ affect the difference $\pi \lambda_{\pi}-\lambda$ between the expected incentive payment $\pi \lambda_{\pi}$ and the effort cost $\lambda$. If, at the margin, an increase in $\bar{y}$ or $I$ raises $\pi \lambda_{\pi}-\lambda$, then, in the optimal contract under consideration, the variable in question is fixed at a value that is inefficiently low in the sense that a small increase would raise the aggregate surplus. If an increase in $\bar{y}$ or $I$ lowers the difference $\pi \lambda_{\pi}-\lambda$, the variable in question is fixed at a value that is inefficiently high in the sense that a small decrease would raise the aggregate surplus.

Which of these possibilities arises depends on the technology. I first consider the Cobb-Douglas specification

$$
f(I, \ell, \pi)=X(\pi) g(I, \ell)=X(\pi) I^{a} \ell^{b},
$$

where $X(\cdot)$ is again decreasing and concave, $a$ and $b$ are positive, and $a+b<$ 1. For this specification, one has

$$
\lambda(\bar{y}, I, \pi)=\left(\frac{\bar{y}}{X(\pi) I^{a}}\right)^{\frac{1}{b}}
$$


and

$$
\lambda_{\bar{y}}=\frac{\lambda}{b \bar{y}}, \lambda_{I}=-\frac{a \lambda}{b I}, \lambda_{\pi}=\frac{\left(-X^{\prime}\right)}{b X} \lambda .
$$

The difference between the expected incentive payment and the effort cost is given as

$$
\pi \lambda_{\pi}-\lambda=\left(\frac{\left(-\pi X^{\prime}\right)}{b X}-1\right) \lambda,
$$

which, for given $\pi$, is just proportional to $\lambda$. Conditions (3.11) and (3.12) can therefore be rewritten as

$$
\pi-\lambda_{\bar{y}}=K \lambda_{\bar{y}}
$$

and

$$
-\lambda_{I}-1=K \lambda_{I}
$$

where

$$
K:=\frac{\mu-1}{\mu}\left(\frac{\left(-\pi X^{\prime}\right)}{b X}-1\right) .
$$

The wedges between the marginal benefits and and the marginal effort costs of increasing $\bar{y}$ and $I$ are proportional to each other and have the same sign. The following result shows that these wedges are in fact positive. Investment and effort are below their surplus maximizing levels. They are even below the levels $\hat{I}(\pi)$ and $\hat{\ell}(\pi)$ that maximize surplus when $\pi$ is taken as given.

Proposition 3.6 Assume that $\tilde{\theta} \equiv 1$. If $f$ takes the form (3.23) and if $A<I^{*}$, then, any optimal contract satisfies

$$
I<\hat{I}(\pi) \text { and } \ell<\hat{\ell}(\pi)
$$

where, for any $\rho$,

$$
(\hat{I}(\rho), \hat{\ell}(\rho)):=\arg \max _{(\hat{I}, \hat{\ell})}\left[\rho X(\rho) \hat{I}^{a} \hat{\ell}^{b}-\hat{\ell}-\hat{I}\right] .
$$

Proof. By routine calculations, conditions (3.27) and (3.28) yield

$$
\begin{gathered}
\bar{y}=X(\pi)\left[\frac{(\pi X(\pi))^{a+b} a^{a} b^{b}}{1+K}\right]^{\frac{1}{1-a-b}}, \\
I=\left[\frac{\pi X(\pi) a^{1-b} b^{b}}{(1+K)^{b}}\right]^{\frac{1}{1-a-b}},
\end{gathered}
$$


and

$$
\ell=\lambda(\bar{y}, I, \pi)=\left[\frac{\pi X(\pi) a^{a} b^{1-a}}{(1+K)^{1-a}}\right]^{\frac{1}{1-a-b}} .
$$

By (3.26) and (3.29), one has

$$
K=\frac{\mu-1}{\mu} \frac{\pi \lambda_{\pi}-\lambda}{\lambda} .
$$

By Lemma 3.4, $A<I^{*}$ implies $\mu>1$. By Lemma 2.1 and (2.18), $\pi \lambda_{\pi}-\lambda>$ $A \geq 0$. The constant $K$ is therefore positive, and equations (3.33) and (3.34) yield

$$
I<\left[\pi X(\pi) a^{1-b} b^{b}\right]^{\frac{1}{1-a-b}}
$$

and

$$
\ell<\left[\pi X(\pi) a^{a} b^{1-a}\right]^{\frac{1}{1-a-b}} .
$$

These inequalities are equivalent to (3.30).

Because $\pi<\pi^{*}$ and $\pi X(\pi)<\pi^{*} X\left(\pi^{*}\right)$, the pair $(\hat{I}(\pi), \hat{\ell}(\pi))$ is strictly smaller than the first-best pair $\left(I^{*}, \ell^{*}\right)$. Trivially, therefore, Proposition 3.6 also implies $I<I^{*}$ and $\ell<\ell^{*}$.

Despite the apparent symmetry of results about investment and effort, Proposition 3.6 should be interpreted as an undereffort result rather than an underinvestment result. Underinvestment arises only because there is too little effort and, in ths Cobb-Douglas specification, the marginal return to investment depends positively on $\ell$. Conditional on $\ell$ and $\pi$, the investment level in an optimal contract for the Cobb-Douglas specification is in fact efficient. By contrast, conditional on $I$ and $\pi$, the effort level is inefficiently low.

Proposition 3.7 Assume that $\tilde{\theta} \equiv 1$. If $f$ takes the form (3.23) and if $A<I^{*}$, then, any optimal contract satisfies

$$
I=\arg \max _{\hat{I}}\left[\pi X(\pi) \hat{I}^{a} \ell^{b}-\ell-\hat{I}\right]
$$

and

$$
\ell<\arg \max _{\ell}\left[\pi X(\pi) I^{a} \hat{\ell}^{b}-\hat{\ell}-I\right]
$$

Moreover

$$
\frac{I}{\ell}>\frac{I^{*}}{\ell^{*}}
$$


Proof. By standard calculations, one has

$$
\arg \max _{\hat{I}}\left[\pi X(\pi) \hat{I}^{a} \ell^{b}-\ell-\hat{I}\right]=\left[a \pi X(\pi) \ell^{b}\right]^{\frac{1}{1-a}} .
$$

Upon using (3.34) to substitute for $\ell$ and simplifying, one finds that this is equal to the right-hand side of (3.33). One also has

$$
\arg \max _{\ell}\left[\pi X(\pi) I^{a} \hat{\ell}^{b}-\hat{\ell}-I\right]=\left[b \pi X(\pi) I^{a}\right]^{\frac{1}{1-b}} .
$$

Upon using (3.33) to substitute for $I$ and rearranging terms, using (3.34), one obtains

$\arg \max _{\ell}\left[\pi X(\pi) I^{a} \hat{\ell}^{b}-\hat{\ell}-I\right]=(1+K)^{\frac{1}{1-b}}\left[\frac{\pi X(\pi) a^{a} b^{1-a}}{(1+K)^{1-a}}\right]^{\frac{1}{1-a-b}}=(1+K)^{\frac{1}{1-b}} \ell$,

which proves (3.39). For (3.40), it suffices to note that (3.33) and (3.34) imply

$$
\frac{I}{\ell}=(1+K) \frac{a}{b}=(1+K) \frac{I^{*}}{\ell^{*}} .
$$

If one compares Propositions 3.6 and 3.7 with Propositions 3.5 and 3.2, one sees that there are two distinct undereffort results, which correspond to the two dimensions of returns to effort, the success probability $\pi$ and the conditional return expectation $\bar{y}$. Propositions 3.5 and 3.2 assert that, at an optimal contract, additional effort devoted to raising $\pi$ would raise expected surplus. Propositions 3.6 and 3.7 assert that, at an optimal contract, additional effort devoted to raising $\bar{y}$ would raise expected surplus.

The underlying reasons for the two undereffort results are different. Both are due to the fact that $\pi$ is not observable and that an increase in effort to raise $\pi$ or $\bar{y}$ raises the requisite incentive payment $\pi \lambda_{\pi}$ by more than it raises $\lambda$. However, for an increase in effort devoted to raising $\pi$, this wedge arises naturally from the convexity of the effort cost function. For an increase in effort devoted to raising $\bar{y}$, the wedge arises from the particular mathematical structure of the effort cost function in the Cobb-Douglas case. Because $\lambda_{\bar{y}}=\frac{\lambda}{b \bar{y}}$, the inequality $\pi \lambda_{\bar{y} \pi}-\lambda_{\bar{y}}>0$ is directly implied by the inequality $\pi \lambda_{\pi}-\lambda>0$. 


\subsection{The Role of the Elasticity of Substitution}

The Cobb-Douglas specification (3.23) is very special. Therefore, it is of interest to see which of the preceding results are robust to changes in the specification of the technology. For this purpose, I will consider the more general separable homogeneous specification

$$
f(I, \ell, \pi)=X(\pi) g(I, \ell)=X(\pi) \psi(I, \ell)^{\beta},
$$

where $\psi$ is linearly homogeneous and $0<\beta<1 .{ }^{13}$ I will be particularly interested in the role of the elasticity of substitution between investment and effort, $\sigma=\frac{\psi_{\ell} \psi_{I}}{\psi \psi_{\ell I}}$. In the general case of (3.41), this elasticity depends on the investment-effort pair that is chosen. However, for the CES specification

$$
f(I, \ell, \pi)=X(\pi)\left[a^{1-\alpha} I^{\alpha}+(1-a)^{1-\alpha} \ell^{\alpha}\right]^{\frac{\beta}{\alpha}},
$$

with $\alpha<1, \alpha \neq 0$, and $0<\beta<1$, it is an exogenous constant,

$$
\sigma=\frac{1}{1-\alpha}
$$

In the Cobb-Douglas specification (3.23), of course, the elasticity of substitution is also constant and equal to one.

For the separable homogeneous specification (3.41), (3.18) takes the form

$$
\lambda_{\bar{y}}=\frac{1}{\beta X \psi^{\beta-1} \psi_{\ell}}, \lambda_{I}=-\frac{\psi_{I}}{\psi_{\ell}}, \lambda_{\pi}=-\frac{X^{\prime}}{\beta X} \frac{\psi}{\psi_{\ell}} .
$$

The difference between the expected incentive payment $\pi \lambda_{\pi}$ and the effort $\operatorname{cost} \lambda$ is therefore computed as

$$
\pi \lambda_{\pi}-\lambda=\left(\frac{r(\pi)}{\beta} \frac{\psi}{\psi_{\ell} \lambda}-1\right) \lambda
$$

where

$$
r(\pi):=\frac{-\pi X^{\prime}(\pi)}{X(\pi)}
$$

Whereas, in the Cobb-Douglas case, the ratio $\frac{\psi}{\psi_{\ell} \lambda}$ is a constant and plays no role in assessing the effects of changes in $\bar{y}$ or $I$ on the difference $\pi \lambda_{\pi}-\lambda$,

\footnotetext{
${ }^{13}$ As a special case of the yet more general homothetic specification $g(I, \ell)=h(\psi(I, \ell))$, the homogeneous specification (3.41) involves a constant ratio $h(\psi) / h^{\prime}(\psi) \psi$. As shown in Hellwig (1994), the results presented here generalize to the case where $h(\psi) / h^{\prime}(\psi) \psi$ is nondecreasing in $\psi$.
} 
in the general homogeneous case, the ratio $\frac{\psi}{\psi_{\ell} \lambda}$ depends on $\bar{y}$ and $I$, as well as $\pi$. Changes in $\bar{y}$ or $I$ affect the difference $\pi \lambda_{\pi}-\lambda$ through their effects on this ratio, as well as their effects on $\lambda$. The first-order conditions (3.11) and (3.12) for $\bar{y}$ and $I$ now take the form:

$$
\pi-\lambda_{\bar{y}}=\frac{\mu-1}{\mu}\left[\frac{\pi \lambda_{\pi}-\lambda}{\lambda}+\frac{r(\pi)}{\beta} \frac{\psi_{I} I}{\psi_{\ell} \ell} \frac{1-\sigma}{\sigma}\right] \lambda_{\bar{y}}
$$

and

$$
-\lambda_{I}-1=\frac{\mu-1}{\mu}\left[\frac{\pi \lambda_{\pi}-\lambda}{\lambda}+\frac{r(\pi)}{\beta} \frac{\psi}{\psi_{\ell} \ell} \frac{1-\sigma}{\sigma}\right] \lambda_{I} .
$$

The first terms on the right-hand sides of (3.47) and (3.48) correspond to the wedge $K=\frac{\mu-1}{\mu} \frac{\pi \lambda_{\pi}-\lambda}{\lambda}$ in the Cobb-Douglas case; these terms reflect the fact that, for a given value of the ratio $\frac{\psi}{\psi_{\ell} \lambda}$, the change in $\lambda$ that is induced by a change in $\bar{y}$ or $I$ is accompanied by an even greater change in the incentive payment $\pi \lambda$. The other terms reflect the effects of changes in $\bar{y}$ or $I$ on the ratio $\frac{\psi}{\psi_{\ell} \lambda}$. At the margin, these effects depend on whether the elasticity of substitution between investment and effort is greater or less than one. This finding can be understood by observing that $\frac{\psi}{\psi_{\ell} \lambda}$ is the inverse of the "share" of effort in the "output" $\psi$ when the "inputs" $\ell$ and $I$ are valued at their marginal products.

Because of the effects of $\bar{y}$ and $I$ on the ratio $\frac{\psi}{\psi_{\ell} \lambda}$ in $(3.45)$, investment in an optimal contract no longer exhibits the conditional-efficiency property of Proposition 3.7 when $\sigma \neq 1$. Instead, one obtains:

Proposition 3.8 Assume that $\tilde{\theta} \equiv 1$. If $f$ takes the separable homogeneous form (3.41) and if $A<I^{*}$, then, any optimal contract satisfies

$$
I \lesseqgtr \arg \max _{\hat{I}}\left[\pi X(\pi) \psi(\hat{I}, \ell)^{\beta}-\ell-\hat{I}\right] \quad \text { as } \quad \sigma \gtreqless 1 .
$$

Proof. Upon multiplying (3.47) by $\frac{\lambda_{I}}{\lambda_{\bar{y}}}$ and subtracting the result from (3.48), using the fact that, by homogeneity, $\psi=\psi_{I} I+\psi_{\ell} \ell$, one obtains

$$
-\pi \frac{\lambda_{I}}{\lambda_{\bar{y}}}-1=\frac{\mu-1}{\mu} \frac{r(\pi)}{\beta} \frac{1-\sigma}{\sigma} \lambda_{I} .
$$

By (3.44), it follows that

$$
\beta \pi X \psi^{\beta-1} \psi_{I}-1=-\frac{\mu-1}{\mu} \frac{r(\pi)}{\beta} \frac{\psi_{I}}{\psi_{\ell}} \frac{1-\sigma}{\sigma}
$$


and, hence, that

$$
\beta \pi X \psi^{\beta-1} \psi_{I} \gtreqless 1 \text { as } \sigma \gtreqless 1 .
$$

The proposition follows immediately.

The left-hand side of equation (3.51) indicates the difference between the expected marginal product of an increase in $I$ and the opportunity cost of the requisite funds when $\ell$ and $\pi$ are taken as given. The right-hand side corresponds to the effect of this increase on the ratio $\frac{\psi}{\psi_{\ell} \lambda}$ in (3.45). If $\sigma>1$, an increase in $I$ and $\bar{y}$, keeping $\ell$ and $\pi$ constant, raises the ratio $\frac{\psi}{\psi_{\ell} \ell}=1+\frac{\psi_{I} I}{\psi_{\ell} \ell}$; if $\sigma<1$, the increase in $I$ lowers this ratio. At an optimal contract, therefore, the expected return from a marginal increase in $I$ is strictly greater than the cost if $\sigma>1$ and less than the cost if $\sigma<1$. Conditional on $\ell$ and $\pi$, investment under an optimal contract is inefficiently low if $\sigma>1$ and inefficiently high if $\sigma<1$. The conditional efficiency of investment in the Cobb-Douglas case thus marks the boundary between the cases $\sigma>1$ and $\sigma<1$.

The corresponding effect in the first-order condition for the return target $\bar{y}$ works in the opposite direction. From (3.47), one finds that

$$
\frac{\pi-\lambda_{\bar{y}}}{\lambda_{\bar{y}}} \gtreqless \frac{\mu-1}{\mu} \frac{\pi \lambda_{\pi}-\lambda}{\lambda} \text { as } \sigma \lesseqgtr 1
$$

Whereas, in the Cobb-Douglas case, the wedge in the condition for $\bar{y}$ was given by $K:=\frac{\mu-1}{\mu} \frac{\pi \lambda_{\pi}-\lambda}{\lambda}$, in the more general specification considered here, the wedge is greater or less than $K$ depending on whether the elasticity of substitution is less than or greater than one. If $\sigma \leq 1$, the conditional undereffort result of Proposition 3.7 is thereby enhanced.

Proposition 3.9 Assume that $\tilde{\theta} \equiv 1$. If $f$ takes the separable homogeneous form (3.41) and if $A<I^{*}$, then, for any optimal contract, one has

$$
\ell<\arg \max _{\hat{\ell}}\left[\pi X(\pi) \psi(I, \hat{\ell})^{\beta}-\hat{\ell}-I\right]
$$

and

$$
\frac{I}{\ell}>\frac{I^{*}}{\ell^{*}}
$$

if $\sigma<1$.

Proof. By the same argument as in the proof of Proposition 3.6, one has $\frac{\mu-1}{\mu} \frac{\pi \lambda_{\pi}-\lambda}{\lambda}>0$. By (3.47), it follows that $\sigma<1$ implies $\frac{\pi-\lambda_{\bar{y}}}{\lambda_{\bar{y}}}>0$, hence, by 
(3.44), $\beta \pi X(\pi) \psi^{\beta-1} \psi_{\ell}-1>0$, which yields (3.54). By (3.48), $\sigma<1$ also implies $\frac{-\lambda_{I}-1}{\lambda_{I}}>0$, hence $\frac{\psi_{\ell}}{\psi_{I}}>1$, which yields $(3.55)$.

For $\sigma>1$, the different effects work in opposite directions. If $\sigma$ is close to one, the inequalities (3.54) and (3.55) will still be valid because the term involving $\sigma-1$ is dominated. The following proposition shows that (3.55) is reversed if $\sigma$ is a sufficiently large constant. The proof of this proposition is given in the Appendix.

Proposition 3.10 Assume that $\tilde{\theta} \equiv 1$. If $f$ takes the separable CES form (3.42) and if $A<I^{*}$, then, for any optimal contract, one has

$$
\frac{I}{\ell}<\frac{I^{*}}{\ell^{*}}
$$

if $\sigma>\max \left(2, \frac{1}{\beta a}\right)$.

Because of the effects of $\sigma$ differing from one, I do not have an analogue of the separate findings of unconditional underinvestment and undereffort that Proposition 3.6 provides for the Cobb-Douglas case. However, the following result shows that the sum of investment and effort and the resulting output are smaller than they would be under surplus maximization.

Proposition 3.11 Assume that $\tilde{\theta} \equiv 1$. If $f$ takes the separable homogeneous form (3.41) and if $A<I^{*}$, then, any optimal contract satisfies

$$
I+\ell<\hat{I}(\pi)+\hat{\ell}(\pi) \text { and } \psi(I, \ell)<\psi(\hat{I}(\pi), \hat{\ell}(\pi)),
$$

where, for any $\rho$,

$$
(\hat{I}(\rho), \hat{\ell}(\rho)):=\arg \max _{(\hat{I}, \hat{\ell})}[\rho X(\rho) \psi(\hat{I}, \hat{\ell})-\hat{\ell}-\hat{I}] .
$$

Proof. By (3.44), (3.47) can be rewritten as

$$
\beta \pi X \psi^{\beta-1} \psi_{\ell}-1=\frac{\mu-1}{\mu}\left[\frac{\pi \lambda_{\pi}-\lambda}{\lambda}-\frac{r(\pi)}{\beta} \frac{\psi_{I} I}{\psi_{\ell} \ell} \frac{\sigma-1}{\sigma}\right]
$$

If one multiplies this equation by $\ell=\lambda(\bar{y}, I, \pi)$ and equation (3.51) by $I$ and adds the results up, one obtains

$$
\beta \pi X \psi(I, \ell)^{\beta-1}\left[\psi_{\ell} \ell+\psi_{I} I\right]-I-\ell=K \ell,
$$


where again $K:=\frac{\mu-1}{\mu} \frac{\pi \lambda_{\pi}-\lambda}{\lambda}$. and, by the same argument as in the proof of Proposition 3.6, $K>0 .{ }^{14}$ By the linear homogeneity of $\psi$, it follows that

$$
\beta \pi X \psi(I, \ell)^{\beta}>I+\ell
$$

The first-order conditions for $\hat{I}(\pi)$ and $\hat{\ell}(\pi)$ imply

$$
\beta \pi X \psi(\hat{I}(\pi), \hat{\ell}(\pi))^{\beta-1} \psi_{\ell}=\beta \pi X \psi(\hat{I}(\pi), \hat{\ell}(\pi))^{\beta-1} \psi_{I}=1,
$$

hence

$$
\beta \pi X \psi(\hat{I}(\pi), \hat{\ell}(\pi))^{\beta-1}\left[\psi_{\ell} \hat{\ell}(\pi)+\psi_{I} \hat{I}(\pi)\right]-\hat{\ell}(\pi)-\hat{I}(\pi)=0 .
$$

By the linear homogeneity of $\psi$, it follows that

$$
\beta \pi X \psi(\hat{I}(\pi), \hat{\ell}(\pi))^{\beta}=\hat{I}(\pi)+\hat{\ell}(\pi) .
$$

From (3.60) and (3.62), one obtains

$$
\frac{\psi(I, \ell)^{\beta}}{I+\ell}>\frac{\psi(\hat{I}(\pi), \hat{\ell}(\pi))^{\beta}}{\hat{I}(\pi)+\hat{\ell}(\pi)} .
$$

One also has $\psi(\hat{I}, \hat{\ell})=\varphi(\hat{I}(\pi)+\hat{\ell}(\pi))$ and $\psi(I, \ell) \leq \varphi(I+\ell)$, where, for any $R, \varphi(R):=\max _{I} \psi(I, R-I)$. Thus, (3.63) implies

$$
\frac{\varphi(I+\ell)^{\beta}}{I+\ell}>\frac{\varphi(\hat{I}(\pi)+\hat{\ell}(\pi))^{\beta}}{\hat{I}(\pi)+\hat{\ell}(\pi)} .
$$

Given that $\psi$ is linearly homogenous and $0<\beta<1$, the function $R \rightarrow$ $\varphi(R)^{\beta}$ is strictly increasing and strictly concave. Therefore (3.64) implies $I+\ell<\hat{I}(\pi)+\hat{\ell}(\pi)$ and $\varphi(I+\ell)<\varphi(\hat{I}(\pi)+\hat{\ell}(\pi))$. Since $\psi(I, \ell) \leq \varphi(I+\ell)$ and $\psi(\hat{I}, \hat{\ell})=\varphi(\hat{I}(\pi)+\hat{\ell}(\pi))$, the proposition is thereby proved.

By the same logic as in the Cobb-Douglas case, Proposition 3.11 also implies that $I+\ell<I^{*}+\ell^{*}$ and $\psi(I, \ell)<\psi\left(I^{*}, \ell^{*}\right)$. Upon combining these findings with Propositions 3.9 and 3.10, one also obtains:

Corollary 3.12 If $\sigma<1$, the optimal contract in Proposition 3.9 also satisfies $\ell<\hat{\ell}(\pi)<\ell^{*}$. If $f$ takes the separable CES form (3.42) and if $\sigma>\max \left(2, \frac{1}{\beta a}\right)$, the optimal contract satisfies $I<\hat{I}(\pi)<I^{*}$.

\footnotetext{
${ }^{14}$ Note that the left-hand side of (3.59) is the derivative of the function $\delta \rightarrow$ $\pi X(\pi) \psi(\delta I, \delta \ell)^{\beta}-\delta I-\delta \ell$ at the point $\delta=1$. A proportional increase in both, $I$ and $\ell$, would thus raise expected surplus.
} 


\subsection{The Case of Nearly Perfect Substitutes}

If effort and investment are perfect substitutes, the investment-effort mix is a matter of indifference. In this case, a first-best outcome can be achieved without recourse to outside finance, with entrepreneurial effort substituting for investment. A first-best outcome can also be achieved with outside financing of investment sufficient to substitute for the entrepreneur's effort. Both arrangements are incentive-compatible, the first one because there is no outside finance, the second one because the entrepreneur need not be given any incentives. ${ }^{15}$

Given that a first-best outcome can be implemented when effort and investment are perfect substitutes, it seems plausible that payoffs close to first-best should be attainable when effort and investment are imperfect substitutes provided the elasticity of substitution is large. For the separable CES specification (3.42), the following result shows that this intuition is justified. If the elasticity of substitution is large, an optimal contract will be close to the contract with efficient effort and zero outside finance that is first-best when investment and effort are perfect substitutes. By contrast, optimal contracts are far from the contract with zero effort and efficient investment that is first-best when investment and effort are perfect substitutes.

The CES specification (3.42) is parametrized so that first-best outcomes are independent of $\sigma{ }^{16}$ Regardless of $\sigma$, first-best investment and effort levels are computed as $^{17} I^{*}=a C^{*}$ and $\ell^{*}=(1-a) C^{*}$, where

$$
C^{*}=\left[\beta \pi^{*} X\left(\pi^{*}\right)\right]^{\frac{1}{1-\beta}} .
$$

The corresponding surplus,

$$
W^{*}=\frac{1-\beta}{\beta} C^{*},
$$

is also independent of $\sigma$. In the limiting case of perfect substitutes, with

$$
f(I, \ell, \pi)=X(\pi)(I+\ell)^{\beta},
$$

\footnotetext{
${ }^{15}$ By contrast, the argument underlying Proposition 2.4 can still be used to show that one cannot implement first-best input combinations that involve outside finance and a positive effort by the entrepreneur.

${ }^{16}$ The Cobb-Douglas specification (3.23) does not conform to this requirement. For this purpose, (3.23) would have to be replaced by the modified Cobb-Douglas specification $f=X(\pi)(I / a)^{a \beta}(\ell /(1-a))^{(1-a) \beta}$.

${ }^{17}$ To see this, observe, first, that the first-order conditions for $I^{*}$ and $\ell^{*}$ imply $\frac{I^{*}}{a}=\frac{\ell^{*}}{1-a}$, hence, $I^{*}=a C^{*}$ and $\ell^{*}=(1-a) C^{*}$ for some $C^{*}$. The value of $C^{*}$ is obtained by maximizing $\pi^{*} X\left(\pi^{*}\right) C^{\beta}-C$ with respect to $C$.
} 
a first-best outcome still satisfies $I+\ell=C^{*}$ and yields the surplus $W^{*}=$ $\frac{1-\beta}{\beta} C^{*}$. However, in this limiting case, any pair $(I, \ell)$ with $I+\ell=C^{*}$ is first-best, in particular, also the pair $\left(A, C^{*}-A\right)$, which does not involve any outside finance.

Proposition 3.13 Assume that $\tilde{\theta} \equiv 1$, let $f$ take the separable CES form (3.42) and suppose that $A<I^{*}$. For any $\sigma$, let $(I(\sigma), \ell(\sigma), \pi(\sigma), \bar{y}(\sigma), w(\cdot \mid \sigma))$ be an optimal contract when the exponent in $\alpha=\frac{\sigma-1}{\sigma}$. As $\sigma$ goes out of bounds, $(I(\sigma), \ell(\sigma))$ converges to $\left(A, C^{*}-A\right), \pi(\sigma)$ converges to $\pi^{*}$, and $\bar{y}(\sigma)$ converges to $X\left(\pi^{*}\right) C^{* \beta}$. The entrepreneur's payoff from the optimal contract converges to $W^{*}+A$.

Proof. For $\sigma>1$, let $\left(I_{0}(\sigma), \ell_{0}(\sigma), \pi_{0}(\sigma), \bar{y}_{0}(\sigma), w_{0}(\cdot \mid \sigma)\right)$ be the optimal contract with zero investment (and zero outside finance). Thus, $I_{0}(\sigma)=0$, $\ell_{0}(\sigma)=\lambda\left(\bar{y}_{0}(\sigma), 0, \pi_{0}(\sigma) \mid \sigma\right)$,

$$
\left(\bar{y}_{0}(\sigma), \pi_{0}(\sigma)\right) \in \arg \max _{\bar{y}, \pi}[\pi \bar{y}+A-\lambda(\bar{y}, 0, \pi \mid \sigma)],
$$

and $w_{0}(y \mid \sigma)=A+y$ for all $y$. From (3.68), one obtains $\pi_{0}(\sigma)=\pi^{*}$,

$$
\bar{y}_{0}(\sigma)=\frac{1}{\beta \pi^{*}}\left[\beta \pi^{*} X\left(\pi^{*}\right)(1-a)^{(1-\alpha) \frac{\beta}{\alpha}}\right]^{\frac{1}{1-\beta}},
$$

and

$$
\ell_{0}(\sigma)=\left[\beta \pi^{*} X\left(\pi^{*}\right)(1-a)^{(1-\alpha) \frac{\beta}{\alpha}}\right]^{\frac{1}{1-\beta}}
$$

The surplus generated by the entrepreneur's project is

$$
W_{0}(\sigma)=\frac{1-\beta}{\beta}\left[\beta \pi^{*} X\left(\pi^{*}\right)(1-a)^{\frac{(1-\alpha) \beta}{\alpha}}\right]^{\frac{1}{1-\beta}},
$$

and the corresponding payoff for the entrepreneur is $W_{0}(\sigma)+A$. As $\sigma$ goes out of bounds and $\alpha=1-\frac{1}{\sigma}$ converges to one, the term $(1-a)^{\frac{(1-\alpha) \beta}{\alpha}}$ in (3.70) converges to one, and $W_{0}(\sigma)$ converges to the first-best surplus $\frac{1-\beta}{\beta}\left[\beta \pi^{*} X\left(\pi^{*}\right)\right]^{\frac{1}{1-\beta}}=W^{*}$.

Let $U^{* *}(\sigma)$ be the entrepreneur's expected payoff from an optimal contract. Because, trivially, the contract $\left(I_{0}(\sigma), \ell_{0}(\sigma), \pi_{0}(\sigma), \bar{y}_{0}(\sigma), w_{0}(\cdot \mid \sigma)\right)$ is incentive-compatible and satisfies the financiers' participation constraint, $U^{* *}(\sigma)$ cannot be less than $W_{0}(\sigma)+A$. It also cannot be greater than the first-best payoff $W^{*}+A$. Given that $W_{0}(\sigma)$ converges to the first-best surplus $W^{*}$ when $\sigma$ becomes large, it follows that

$$
\lim _{\sigma \rightarrow \infty} U^{* *}(\sigma)=W^{*}+A .
$$


From (3.1) and the fact that an optimal contract satisfies the financiers' participation constraint with equality, one has

$$
U^{* *}(\sigma)=\pi(\sigma) \bar{y}(\sigma)-I(\sigma)-\lambda(\bar{y}(\sigma), I(\sigma), \pi(\sigma))+A .
$$

Thus, (3.71) implies

$$
\lim _{\sigma \rightarrow \infty}[\pi(\sigma) \bar{y}(\sigma)-I(\sigma)-\lambda(\bar{y}(\sigma), I(\sigma), \pi(\sigma))]=W^{*}
$$

From (3.73), it follows that, as $\sigma$ becomes large, $\pi(\sigma)$ converges to $\pi^{*}, I(\sigma)+$ $\ell(\sigma)$ converges to $C^{*}$, and $\bar{y}(\sigma)$ converges to $X\left(\pi^{*}\right) C^{* \beta}$.

It is now sufficient to show that $I(\sigma)$ converges to $A$. From the financiers' participation constraint and Lemma 3.4, one has

$$
I(\sigma)=A+\pi(\sigma) \bar{y}(\sigma)-\pi(\sigma) \lambda_{\pi}(\bar{y}(\sigma), I(\sigma), \pi(\sigma)) .
$$

By (3.44) and (3.46),

$$
\begin{aligned}
\pi(\sigma) \lambda_{\pi}(\bar{y}(\sigma), I(\sigma), \pi(\sigma)) & =\frac{r(\pi(\sigma))}{\beta} \frac{\psi(I(\sigma), \ell(\sigma))}{\psi_{\ell}(I(\sigma), \ell(\sigma))} \\
& =\frac{r(\pi(\sigma))}{\beta} \frac{a^{1-\alpha} I(\sigma)^{\alpha}+(1-a)^{1-\alpha} \ell(\sigma)^{\alpha}}{(1-a)^{1-\alpha} \ell(\sigma)^{\alpha}} \ell(\sigma) \\
& =\frac{r(\pi(\sigma))}{\beta}\left(\frac{a}{1-a} \eta(\sigma)^{\alpha}+1\right) \ell(\sigma) \\
& =\frac{r(\pi(\sigma))}{\beta} \frac{\frac{a}{1-a} \eta(\sigma)^{\alpha}+1}{\frac{a}{1-a} \eta(\sigma)+1}(I(\sigma)+\ell(\sigma)),
\end{aligned}
$$

where $\eta(\sigma):=\frac{(1-a)}{a} \frac{I(\sigma)}{\ell(\sigma)}$. As mentioned, the term $I(\sigma)+\ell(\sigma)$ in (3.75) converges to $C^{*}$ as $\sigma$ goes out of bound. The term $r(\pi(\sigma))$ converges to $r\left(\pi^{*}\right)$, which, by (3.46) and Lemma 3.3, is equal to one. By Proposition $3.10, \eta(\sigma)=\frac{\ell^{*}}{I^{*}} \frac{I(\sigma)}{\ell(\sigma)}$ remains bounded between zero and one; therefore,

$$
\lim _{\sigma \rightarrow \infty} \frac{1+\frac{a}{1-a} \eta(\sigma)^{1-\frac{1}{\sigma}}}{1+\frac{a}{1-a} \eta(\sigma)}=1 .
$$

Equation (3.75) thus yields

$$
\lim _{\sigma \rightarrow \infty} \pi(\sigma) \lambda_{\pi}(\bar{y}(\sigma), I(\sigma), \pi(\sigma))=\frac{1}{\beta} C^{*}=\frac{1}{\beta}\left[\beta \pi^{*} X\left(\pi^{*}\right)\right]^{\frac{1}{1-\beta}} .
$$


Because $\pi(\sigma)$ converges to $\pi^{*}$ and $\bar{y}(\sigma)$ converges to $X\left(\pi^{*}\right) C^{* \beta}$, one also has

$\lim _{\sigma \rightarrow \infty} \pi(\sigma) \bar{y}(\sigma)=\pi^{*} X\left(\pi^{*}\right) C^{* \beta}=\pi^{*} X\left(\pi^{*}\right)\left[\beta \pi^{*} X\left(\pi^{*}\right)\right]^{\frac{\beta}{1-\beta}}=\frac{1}{\beta}\left[\beta \pi^{*} X\left(\pi^{*}\right)\right]^{\frac{1}{1-\beta}}$.

The terms $\pi(\sigma) \bar{y}(\sigma)$ and $\pi(\sigma) \lambda_{\pi}(\bar{y}(\sigma), I(\sigma), \pi(\sigma))$ in (3.74) thus converge to the same limit, which proves that $I(\sigma)$ must converge to $A$.

Although the entrepreneur's payoff converges to the first-best payoff when $\sigma$ goes out of bounds, the input pairs $(I(\sigma), \ell(\sigma))$ remain bounded away from the first-best pair $\left(a C^{*},(1-a) C^{*}\right)$. The following corollary to Proposition 3.13 shows that, when $\sigma$ is large, an optimal contract actually involves overeffort relative to $\ell^{*}$ and undereffort relative to the level that would maximize expected surplus when $I(\sigma)$ and $\pi(\sigma)$ are taken as given. The difference reflects the fact that $I(\sigma)$ is less than the first-best level $I^{*}$. When investment and effort are close to perfect substitutes, this shortfall in investment raises the level of effort that would be required for surplus maximization. The contractually chosen effort level takes account of this need and is therefore greater than $\ell^{*}$. However, conditional on $I(\sigma)$ and $\pi(\sigma)$, it is still inefficiently low.

Corollary 3.14 If $\sigma$ is sufficiently large, the optimal contracts in Proposition 3.13 satisfy

$$
\ell^{*}<\ell(\sigma)<\arg \max _{\hat{\ell}}\left[\pi(\sigma) X(\pi(\sigma)) \psi(I(\sigma), \hat{\ell})^{\beta}-\hat{\ell}-I(\sigma)\right] .
$$

Proof. The first inequality in (3.78) follows from the fact that $\ell(\sigma)$ converges to $C^{*}-A>C^{*}-I^{*}=(1-a) C^{*}=\ell^{*}$. To establish the second inequality in (3.78), I note that the first-order condition (3.47) for $\bar{y}$ can be rewritten as

$$
\frac{\pi(\sigma)}{\lambda_{\bar{y}}}-1=\frac{\mu-1}{\mu}\left[\frac{r(\pi(\sigma))}{\beta}+\frac{r(\pi(\sigma))}{\beta} \frac{1}{\sigma} \frac{\psi_{I} I}{\psi_{\ell} \ell}-1\right] .
$$

Because $\pi(\sigma)$ converges to $\pi^{*}$ and, therefore, $r(\pi(\sigma))$ converges to $r\left(\pi^{*}\right)=1$ as $\sigma$ goes out of bounds, one has $r(\pi(\sigma))>\beta$ if $\sigma$ is sufficiently large. The right-hand side of (3.79) is then positive, and one has $\frac{\pi(\sigma)}{\lambda_{\bar{y}}}>1$, or

$$
\beta \pi(\sigma) X(\sigma) \psi(I(\sigma), \ell(\sigma))^{\beta-1} \psi_{\ell}(I(\sigma), \ell(\sigma))>1,
$$

which yields the second inequality in (3.78) 


\subsection{The Case of Nearly Perfect Complements}

When investment and effort are nearly perfect complements, outcomes under optimal contracting are also close to being efficient.

Proposition 3.15 Assume that $\tilde{\theta} \equiv 1$, let $f$ take the separable CES form (3.42) and suppose that $A<I^{*}$. For any $\sigma$, let $(I(\sigma), \ell(\sigma), \pi(\sigma), \bar{y}(\sigma), w(\cdot \mid \sigma))$ be an optimal contract when the exponent is $\alpha=\frac{\sigma-1}{\sigma}$. As $\sigma$ converges to zero, $(I(\sigma), \ell(\sigma))$ converges to $\left(I^{*}, \ell^{*}\right)=\left(a C^{*},(1-a) C^{*}\right), \pi(\sigma)$ converges to $\pi^{*}$, and $\bar{y}(\sigma)$ converges to $X\left(\pi^{*}\right) C^{* \beta}$. The entrepreneur's payoff from the optimal contract converges to $W^{*}+A$.

Proof. As in the proof of Proposition 3.13, let $U^{* *}(\sigma)$ be the entrepreneur's expected payoff from the contract $(I(\sigma), \ell(\sigma), \pi(\sigma), \bar{y}(\sigma), w(\cdot \mid \sigma))$ when the elasticity of substitution between investment and effort is $\sigma$. I claim that, for any $\varepsilon>0$ and any $\sigma$ sufficiently close to zero, one has $U^{* *}(\sigma) \geq \hat{W}(\varepsilon)+A$ where

$$
\hat{W}(\varepsilon):=\max _{C}\left[\pi^{*} X\left(\pi^{*}\right) C^{\beta}-(1+\varepsilon a) C\right] .
$$

To establish this claim, let

$$
\hat{C}(\varepsilon)=\left[\frac{\beta \pi^{*} X\left(\pi^{*}\right)}{(1+\varepsilon a)}\right]^{\frac{1}{1-\beta}} .
$$

be the maximizer in (3.80) and consider the contract $\left(I_{\varepsilon}, \ell_{\varepsilon}, \pi_{\varepsilon}, \bar{y}_{\varepsilon}, w_{\varepsilon}(\cdot \mid \sigma, B)\right)$ with $I_{\varepsilon}=(1+\varepsilon) a \hat{C}(\varepsilon), \ell_{\varepsilon}=(1-a) \hat{C}(\varepsilon), \pi_{\varepsilon}=\pi^{*}, \bar{y}_{\varepsilon}=X\left(\pi^{*}\right) \hat{C}(\varepsilon)^{\beta}$, and $w_{\varepsilon}(y \mid \sigma, B)=A+B$ for $y<\bar{y}_{\varepsilon}, w_{\varepsilon}(y \mid \sigma, B)=A+B+\lambda_{\pi}\left(\bar{y}_{\varepsilon}, I_{\varepsilon}, \pi^{*}\right)$ for $y \geq \bar{y}_{\varepsilon}$, where $B$ is a positive constant. By Lemma 3.1, this contract is incentive-compatible. It provides the financiers with the expected payoff

$$
\pi^{*} X\left(\pi^{*}\right) \hat{C}(\varepsilon)^{\beta}-\pi^{*} \lambda_{\pi}\left(\bar{y}_{\varepsilon}, I_{\varepsilon}, \pi^{*}\right)-I_{\varepsilon}-B .
$$

By the same calculation as in (3.75), using the fact that $r\left(\pi^{*}\right)=1$, expression (3.81) can be rewritten as

$$
\begin{aligned}
& \pi^{*} X\left(\pi^{*}\right) \hat{C}(\varepsilon)^{\beta}-\frac{1}{\beta} \frac{a^{1-\alpha} I_{\varepsilon}^{\alpha}+(1-a)^{1-\alpha} \ell_{\varepsilon}^{\alpha}}{(1-a)^{1-\alpha} \ell_{\varepsilon}^{\alpha}} \ell_{\varepsilon}-I_{\varepsilon}-B \\
= & \pi^{*} X\left(\pi^{*}\right) \hat{C}(\varepsilon)^{\beta}-\frac{1}{\beta} \frac{a(1+\varepsilon)^{\alpha}+(1-a)}{(1-a)}(1-a) \hat{C}(\varepsilon)-(1+\varepsilon) a \hat{C}(\varepsilon)-B \\
= & \frac{1}{\beta} \hat{C}(\varepsilon)\left[1+\varepsilon a-a(1+\varepsilon)^{\alpha}-(1-a)-\beta(1+\varepsilon) a\right]-B \\
= & \frac{1}{\beta} \hat{C}(\varepsilon)\left[a(1+\varepsilon)(1-\beta)-a(1+\varepsilon)^{\alpha}\right]-B .
\end{aligned}
$$


As $\sigma$ converges to zero and $\alpha=\frac{\sigma-1}{\sigma}$ converges to $-\infty,(1+\varepsilon)^{\alpha}$ converges to zero, and the financiers' expected payoffs from the incentive-compatible contracts $\left(I_{\varepsilon}, \ell_{\varepsilon}, \pi_{\varepsilon}, \bar{y}_{\varepsilon}, w_{\varepsilon}(\cdot \mid \sigma, B)\right)$ converge to $\frac{1}{\beta} \hat{C}(\varepsilon) a(1+\varepsilon)(1-\beta)-B$, which is positive if $B<\frac{1}{\beta} \hat{C}(\varepsilon) a(1+\varepsilon)(1-\beta)$. For $B$ satisfying this inequality, therefore, the contract $\left(I_{\varepsilon}, \ell_{\varepsilon}, \pi_{\varepsilon}, \bar{y}_{\varepsilon}, w_{\varepsilon}(\cdot \mid \sigma, B)\right)$ satisfies the financiers' participation constraint with a strict inequality if $\sigma$ is sufficiently small. This finding in turn implies that, if $\sigma$ is sufficiently small, there exists $B(\varepsilon, \sigma)>0$ such that, for $B=B(\varepsilon, \sigma)$, the contract $\left(I_{\varepsilon}, \ell_{\varepsilon}, \pi_{\varepsilon}, \bar{y}_{\varepsilon}, w_{\varepsilon}(\cdot \mid \sigma, B)\right)$ satisfies the financiers' participation constraint with equality. Since $B(\varepsilon, \sigma)>0$, one has $w_{\varepsilon}(\cdot \mid \sigma, B(\varepsilon, \sigma)) \in \mathcal{W}$. For any sufficiently small $\sigma$, therefore, the entrepreneur's expected payoff $U^{* *}(\sigma)$ from an optimal contract cannot be less than the payoff he gets from the contract $\left(I_{\varepsilon}, \ell_{\varepsilon}, \pi_{\varepsilon}, \bar{y}_{\varepsilon}, w_{\varepsilon}(\cdot \mid \sigma, B)\right)$. Because the financiers' participation constraint holds with equality, the entrepreneur appropriates the entire surplus $\hat{W}(\varepsilon)$, and his payoff is $\hat{W}(\varepsilon)+A$. For any $\varepsilon>0$, one therefore has $\hat{W}(\varepsilon)+A \leq U^{* *}(\sigma) \leq W^{*}+A$ for any sufficiently small $\sigma$.

By inspection of $(3.80), \hat{W}(\varepsilon)$ converges to the first-best surplus $W^{*}$ when $\varepsilon$ goes to zero. Because $\hat{W}(\varepsilon)+A \leq U^{* *}(\sigma) \leq W^{*}+A$ for any sufficiently small $\sigma$, it follows that $U^{* *}(\sigma)$ converges to $W^{*}+A$ as $\sigma$ goes to zero. One easily checks that this is only possible if $(I(\sigma), \ell(\sigma))$ converges to $\left(I^{*}, \ell^{*}\right)=\left(a C^{*},(1-a) C^{*}\right), \pi(\sigma)$ converges to $\pi^{*}$, and $\bar{y}(\sigma)$ converges to $X\left(\pi^{*}\right) C^{* \beta}$ as $\sigma$ goes to zero.

The logic behind this result can be understood by looking at the case of perfect complements itself. For this purpose, consider the specification

$$
f(I, \ell, \pi)=X(\pi) \min \left[\left(\frac{I}{a}\right)^{\beta},\left(\frac{\ell}{1-a}\right)^{\beta}\right],
$$

which yields the same first-best outcomes as the CES specification (3.42), namely, $I^{*}=a C^{*}, \ell^{*}=(1-a) C^{*}, \bar{y}^{*}=X\left(\pi^{*}\right) C^{* \beta}$, with $C^{*}$ given by (3.65). When $f$ is given by (3.82), the effort cost function $\lambda$ is given by

$$
\lambda(\bar{y}, I, \pi)=(1-a)\left(\frac{\bar{y}}{X(\pi)}\right)^{\frac{1}{\beta}} \text { if }\left(\frac{I}{a}\right)^{\beta} \geq \frac{\bar{y}}{X(\pi)} .
$$

If $\left(\frac{I}{a}\right)^{\beta}<\frac{\bar{y}}{X(\pi)}, \lambda(\bar{y}, I, \pi)$ is undefined because, for the given $I$ and $\pi$, the value of $f$ cannot be raised to $\bar{y}$.

The first-best outcome lies on the boundary of the domain of the effort cost function. At this point, the effort cost function has a left-hand partial 
derivative

$$
\lambda_{\pi}\left(\bar{y}^{*}, I^{*}, \pi^{*}-\right)=\frac{1}{\beta}\left(\frac{-X^{\prime}\left(\pi^{*}\right)}{X\left(\pi^{*}\right)}\right) \lambda\left(\bar{y}^{*}, I^{*}, \pi^{*}\right),
$$

but no right-hand derivative. For given $\bar{y}=\bar{y}^{*}$ and $I=I^{*}$, setting $\pi=\pi^{*}$ now maximizes the entrepreneur's payoff $\pi \bar{w}\left(\bar{y}^{*}\right)+(1-\pi) w(0)-\lambda\left(\bar{y}^{*}, I^{*}, \pi\right)$ if and only if

$$
\bar{w}\left(\bar{y}^{*}\right)-w(0) \geq \lambda_{\pi}\left(\bar{y}^{*}, I^{*}, \pi^{*}-\right) .
$$

Thus, in the case of perfect complements, the incentive compatibility condition for the risk choice $\pi$ is an inequality, rather than an equation. This inequality provides enough leeway to eliminate the conflict between incentive compatibility and first-best efficiency that was the subject of Proposition 2.4 .

To see this, consider an incentive scheme satisfying $w(y)=A$ if $y<$ $\bar{y}^{*}$ and $w(y)=A+\bar{y}^{*}-\frac{I^{*}}{\pi^{*}}$ if $y \geq \bar{y}^{*}$. This incentive scheme provides financiers with the payment $\frac{I^{*}}{\pi^{*}}$ if the entrepreneur's project succeeds. They are therefore happy to put up the requisite funds for the investment $I^{*}$. I claim that, in the absence of noise, with $\bar{w}(\cdot)=w(\cdot)$, this incentive scheme also satisfies the incentive compatibility condition (3.85). Because $\bar{y}^{*}=$ $X\left(\pi^{*}\right) C^{* \beta}, I^{*}=a C^{*}$, and $C^{*}=\left[\beta \pi^{*} X\left(\pi^{*}\right)\right]^{\frac{1}{1-\beta}}$, one computes

$$
\begin{aligned}
w\left(\bar{y}^{*}\right)-w(0) & =\frac{1}{\pi^{*}}\left[\pi^{*} X\left(\pi^{*}\right) C^{* \beta}-a C^{*}\right]=\frac{1}{\pi^{*}}\left(\frac{1}{\beta}-a\right) C^{*} \\
& =\frac{1}{\pi^{*}}\left(\frac{1-a}{\beta}+\frac{1-\beta}{\beta} a\right) C^{*}=\frac{1}{\pi^{*}}\left(\frac{1}{\beta} \ell^{*}+\frac{1-\beta}{\beta} I^{*}(\beta .86)\right.
\end{aligned}
$$

Because $\ell^{*}=\lambda\left(\bar{y}^{*}, I^{*}, \pi^{*}\right)$ and, by the first-order condition for $\pi^{*}$, one has $\frac{1}{\pi^{*}}=\frac{-X^{\prime}\left(\pi^{*}\right)}{X\left(\pi^{*}\right)},(3.86)$ and (3.84) yield $\bar{w}\left(\bar{y}^{*}\right)-w(0)>\lambda_{\pi}\left(\bar{y}^{*}, I^{*}, \pi^{*}-\right)$. The incentive compatibility condition (3.85) holds with a strict inequality.

\section{Does Noise Make a Difference?}

If the random variable $\tilde{\theta}$ is nondegenerate, the observation of the realized return in the event of success does not permit the financiers to determine precisely whether $\ell$ and $\pi$ have been chosen so as to provide for the stipulated $\bar{y}$. The observation of $\tilde{y}$ still provides some information about $\bar{y}$, but this information is not perfect. The question is what difference this noise in $\tilde{y}$ makes for optimal contracting.

The distribution of $\tilde{\theta}$ determines the relation between the incentive scheme $w(\cdot)$ and the conditional expectation $\bar{w}(\bar{y})$ of the incentive payment $w(\tilde{y})=$ 


\section{FIGURE 1}

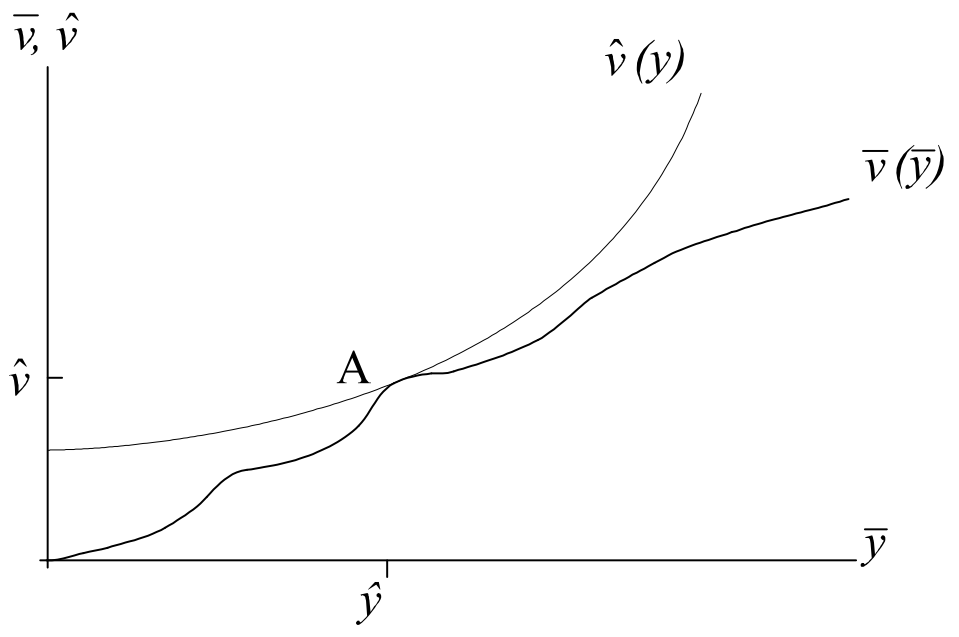

$w(\tilde{\theta} \bar{y})$ when the entrepreneur has chosen effort and risk so that $f(I, \ell, \pi)=$ $\bar{y}$. This matters for the incentive compatibility condition that the entrepreneur should prefer the stipulated $\bar{y}$ over any alternative $\bar{y}^{\prime}$. If $\tilde{\theta} \equiv 1$, one has $w\left(\bar{y}^{\prime}\right)=\bar{w}\left(\bar{y}^{\prime}\right)$, and a forcing contract ensures that this incentive condition is trivially satisfied. If $\tilde{\theta}$ is nondegenerate, a forcing device is no longer available. The question then is whether another incentive scheme can serve the same purpose. ${ }^{18}$

The question is illustrated in Figure 1. To implement a given outcome $(\hat{I}, \hat{\ell}, \hat{\pi}, \hat{y})$, the excess $\bar{w}(\hat{y})-w(0)$ of the entrepreneur's conditional payoff expectation in the event of success over his payoff in the event of failure must take the value $\hat{v}=\lambda_{\pi}(\hat{y}, \hat{I}, \hat{\pi})$. The smooth curve $\hat{v}(\cdot)$ in Figure 1 represents the entrepreneur's indifference curve through the point $(\hat{y}, \hat{v})$, i.e. the locus of all points $(\bar{y}, \bar{v})$ for which $U^{*}(\bar{y}, \bar{v}, \hat{I})=U^{*}(\hat{y}, \hat{v}, \hat{I})$. The question is whether one can find an incentive scheme $w(\cdot)$ such that

$$
\bar{w}(\hat{y})=\int w(\hat{y} \theta) d F(\theta)=w(0)+\hat{v}
$$

\footnotetext{
${ }^{18}$ For a systematic discussion of this kind of problem, see Caillaud, Guesnerie and Rey (1992) and the references given there. Because I require that $w(y) \geq 0$ for all $y$, I cannot rely on their results.
} 
and

$$
\bar{w}(\bar{y})=\int w(\bar{y} \theta) d F(\theta) \leq w(0)+\hat{v}(\bar{y})
$$

for all $\bar{y} \neq \hat{y}$, so that, as shown in the figure, the graph of the function $\bar{v}(\cdot):=\bar{w}(\cdot)-w(0)$ lies nowhere above the entrepreneur's indifference curve through $(\hat{y}, \hat{v})$ and touches it in $(\hat{y}, \hat{v})$ itself. In the case $\tilde{\theta} \equiv 1$, the answer to this question is given by the thinly drawn step function in the figure. In the general case, the question is whether the smoothing that is induced by noise leaves enough scope for adapting the slope and curvature of the function $\bar{v}(\cdot)$ to the requirements of incentive compatibility. To investigate this, I first consider the indifference curve $\hat{v}(\cdot)$.

Lemma 4.1 If $f$ takes the separable form (3.16), the slope of the indifference curve $\hat{v}(\cdot)$ is given by

$$
\frac{d \hat{v}}{d \bar{y}}(\bar{y})=\frac{1}{r(\bar{\pi}(\bar{y}, \hat{v}(\bar{y}), \hat{I}))} \frac{\hat{v}(\bar{y})}{\bar{y}},
$$

where, for any $\bar{y}, \bar{v}$, and $I, \bar{\pi}(\bar{y}, \bar{v}, I):=\arg \max _{\pi}[\pi \bar{v}-\lambda(\bar{y}, I, \pi)]$.

If, in addition, the effort cost function $\lambda$ satisfies

$$
\pi \lambda_{\pi \bar{y}}(\bar{y}, I, \pi)-\lambda_{\bar{y}}(\bar{y}, I, \pi) \geq 0
$$

for all $(\bar{y}, I, \pi)$ for which $\pi \lambda_{\pi}-\lambda>0$, the elasticity $\frac{\bar{y}}{\hat{v}(\bar{y})} \frac{d \hat{v}}{d \bar{y}}(\bar{y})$ of the entrepreneur's indifference curve through any point $\left(\hat{y}, \lambda_{\pi}(\hat{y}, \hat{I}, \hat{\pi})\right)$ with $\hat{\pi} \lambda_{\pi}(\hat{y}, \hat{I}, \hat{\pi})-$ $\lambda(\hat{y}, \hat{I}, \hat{\pi})>0$ is a nondecreasing function of $\bar{y}$.

Proof. For any $\bar{y}$ and $\bar{v},(2.18)$ implies

$$
U^{*}(\bar{y}, \bar{v}, \hat{I})=\bar{\pi}(\bar{y}, \bar{v}, \hat{I}) \bar{v}-\lambda(\bar{y}, I, \bar{\pi}(\bar{y}, \bar{v}, \hat{I})),
$$

where $\bar{\pi}(\bar{y}, \bar{v}, \hat{I}):=\arg \max _{\pi}[\pi \bar{v}-\lambda(\bar{y}, \hat{I}, \pi)]$. The indifference curve $\hat{v}(\cdot)$ is thus implicitly defined by the equation

$$
\bar{\pi}(\bar{y}, \hat{v}(\bar{y}), \hat{I}) \hat{v}(\bar{y})-\lambda(\bar{y}, \hat{I}, \bar{\pi}(\bar{y}, \hat{v}(\bar{y}), \hat{I}))=U^{*}(\hat{y}, \hat{v}, \hat{I}) .
$$

By the implicit function theorem and the envelope theorem, therefore,

$$
\frac{d \hat{v}}{d \bar{y}}(\bar{y})=-\frac{U_{\bar{y}}^{*}(\bar{y}, \hat{v}(\bar{y}), \hat{I})}{U_{v}^{*}(\bar{y}, \hat{v}(\bar{y}), \hat{I})}=\frac{\lambda_{\bar{y}}(\bar{y}, \hat{I}, \bar{\pi}(\bar{y}, \hat{v}(\bar{y}), \hat{I}))}{\bar{\pi}(\bar{y}, \hat{v}(\bar{y}), \hat{I})} .
$$

For the separable specification (3.16), with

$$
\lambda_{\bar{y}}=\frac{1}{X g_{\ell}}=-\frac{\lambda_{\pi}}{X^{\prime} g}=-\frac{X}{X^{\prime}} \frac{\lambda_{\pi}}{\bar{y}}
$$


it follows that

$$
\frac{d \hat{v}}{d \bar{y}}(\bar{y})=\frac{1}{r(\bar{\pi}(\bar{y}, \hat{v}(\bar{y}), \hat{I}))} \frac{\lambda_{\pi}(\bar{y}, \hat{I}, \bar{\pi}(\bar{y}, \hat{v}(\bar{y}), \hat{I}))}{\bar{y}},
$$

where, as before, for any $\pi, r(\pi)=\frac{-\pi X^{\prime}(\pi)}{X(\pi)}$. By Lemma 2.3, this yields (4.3).

From (4.5) and the first-order condition $\hat{v}(\bar{y})=\lambda_{\pi}(\bar{y}, \hat{I}, \bar{\pi}(\bar{y}, \hat{v}(\bar{y}), \hat{I}))$, one computes

$$
\bar{\pi} \lambda_{\pi}-\lambda=U^{*}(\bar{y}, \hat{v}(\bar{y}), \hat{I})=\hat{\pi} \lambda_{\pi}(\hat{y}, \hat{I}, \hat{\pi})-\lambda(\hat{y}, \hat{I}, \hat{\pi})
$$

and

$$
\frac{d}{d \bar{y}} \bar{\pi}(\bar{y}, \hat{v}(\bar{y}), \hat{I})=-\frac{\bar{\pi} \lambda_{\pi \bar{y}}-\lambda_{\bar{y}}}{\bar{\pi} \lambda_{\pi \pi}}
$$

where $\bar{\pi}=\bar{\pi}(\bar{y}, \hat{v}(\bar{y}), \hat{I})$, and $\lambda_{\pi}, \lambda, \lambda_{\pi \bar{y}}, \lambda_{\bar{y}}$, and $\lambda_{\pi \pi}$ are all evaluated at $(\bar{y}, I, \bar{\pi})$. Under the premises of the second statement of the lemma, (4.9) yields $\bar{\pi} \lambda_{\pi}-\lambda>0$ and, therefore, $\bar{\pi} \lambda_{\pi \bar{y}}-\lambda_{\bar{y}} \geq 0$. By (4.10), it follows that $\bar{\pi}(\bar{y}, \hat{v}(\bar{y}), \hat{I})$ is nonincreasing in $\bar{y}$. Under the given monotonicity and curvature assumptions on $f$, and, thereby, on $X(\cdot)$, the ratio $r(\pi)=$ $\frac{-\pi X^{\prime}(\pi)}{X(\pi)}$ is everywhere increasing in $\pi$. Under the given assumptions, therefore, $r(\bar{\pi}(\bar{y}, \hat{v}(\bar{y}), \hat{I}))$ is nonincreasing in $\bar{y}$. The second statement of the lemma follows from (4.3).

\subsection{A Bothersome Result}

Lemma 4.1 and Proposition 3.5 imply that, at $\bar{y}=\hat{y}$, the elasticity of the indifference curve $\hat{v}(\cdot)$ is equal to $\frac{1}{r(\hat{\pi})}$ and is strictly greater than one. If the distribution of the random variable $\tilde{\theta}$ is very flat, so to say, "close to a uniform distribution on $\mathbb{R}_{+}$", it may not be possible to bring the slope of $\bar{v}(\cdot)$ at $\hat{y}$ to this level of steepness. For such distributions, the elasticity of the function $\bar{y} \rightarrow \bar{w}(\bar{y})=E w(\tilde{\theta} \bar{y})$ may be bounded, uniformly for all incentive schemes $w(\cdot) \in \mathcal{W}$. If the bound on the elasticity of $\bar{w}(\cdot)$ is too small, the outcome $(\hat{I}, \hat{\ell}, \hat{\pi}, \hat{y})$ cannot be implemented by any incentive scheme.

Proposition 4.2 Assume that $f$ takes the separable form (3.16) and that $A<I^{*}$. Let $(\hat{I}, \hat{\ell}, \hat{\pi}, \hat{y}, \hat{w}(\cdot))$ be an optimal contract for the case $\tilde{\theta} \equiv 1$. For $k>1$, let $\bar{\theta}(k)=2(k-1) / k$, and assume that the distribution of $\tilde{\theta}$ takes the form

$$
F(\theta)=\frac{k}{k+1} \frac{\theta}{\bar{\theta}(k)} \quad \text { if } \theta \leq \bar{\theta}(k)
$$




$$
F(\theta)=1-\frac{1}{k+1}\left(\frac{\bar{\theta}(k)}{\theta}\right)^{k} \quad \text { if } \theta>\bar{\theta}(k) .
$$

If $k \in\left(1, \frac{1}{r(\hat{\pi})}\right)$, there exists no incentive scheme $w(\cdot) \in \mathcal{W}$ that implements the outcome $(\hat{I}, \hat{\ell}, \hat{\pi}, \hat{y})$ and satisfies the financiers' participation constraint when $\tilde{\theta}$ has the distribution $F$ that is given by (4.11) and (4.12).

Proof. Suppose that, for some $k \in\left(1, \frac{1}{r(\hat{\pi})}\right)$, the outcome $(\hat{I}, \hat{\ell}, \hat{\pi}, \hat{y})$ is implemented by an incentive scheme $w(\cdot) \in \mathcal{W}$. As discussed in the text, let $\hat{v}=\hat{w}(\hat{y})-\hat{w}(0)$ and let $\hat{v}(\cdot)$ be the entrepreneur's indifference curve through $(\hat{y}, \hat{v})$. For $\bar{v}(\cdot)=\bar{w}(\cdot)-w(0)$, the incentive compatibility conditions $(2.21)$ and (2.22) require that

$$
\bar{v}(\hat{y})=\lambda_{\pi}(\hat{y}, \hat{I}, \hat{\pi})=\hat{v}
$$

and

$$
\frac{d \hat{v}}{d \bar{y}}(\hat{y})=\frac{d \bar{v}}{d \bar{y}}(\hat{y})
$$

For the function $\bar{v}(\cdot)$, one computes

$$
\begin{aligned}
\bar{v}(\bar{y}) & =\int_{0}^{\bar{\theta}(k)} w(\theta \bar{y}) \frac{k}{k+1} \overline{\bar{\theta}(k)} d \theta+\int_{\bar{\theta}(k)}^{\infty} w(\theta \bar{y}) \frac{k}{k+1} \frac{\bar{\theta}(k)^{k}}{\theta^{k+1}} d \theta-w(0) \\
& =\int_{0}^{\bar{\theta}(k) \bar{y}} w(x) \frac{k}{k+1} \frac{1}{\bar{\theta}(k) \bar{y}} d x+\int_{\bar{\theta}(k) \bar{y}}^{\infty} w(x) \frac{k}{k+1} \frac{\bar{\theta}(k)^{k} \bar{y}^{k}}{x^{k+1}} d x-w(0)
\end{aligned}
$$

Because the density of $F$ is continuous and, for $w(\cdot) \in \mathcal{W}, w(x) \geq 0$ for all $x$, one obtains

$$
\begin{aligned}
\frac{d \bar{v}}{d \bar{y}}(\hat{y}) & =-\int_{0}^{\bar{\theta}(k) \hat{y}} w(x) \frac{k}{k+1} \frac{1}{\bar{\theta}(k) \hat{y}^{2}} d x+\int_{\bar{\theta}(k) \hat{y}}^{\infty} w(x) \frac{k^{2}}{k+1} \frac{\bar{\theta}(k)^{k} \hat{y}^{k-1}}{x^{k+1}} d x \\
& \leq \frac{k}{\hat{y}} \int_{\bar{\theta}(k) \bar{y}}^{\infty} w(x) \frac{k}{k+1} \frac{\bar{\theta}(k)^{k} \hat{y}^{k}}{x^{k+1}} d x \leq k \frac{[\bar{v}(\hat{y})+w(0)]}{\hat{y}} \\
& <\frac{1}{r(\hat{\pi})} \frac{[\bar{v}(\hat{y})+w(0)]}{\hat{y}} .
\end{aligned}
$$

Since, trivially, $\hat{\pi}=\bar{\pi}(\hat{y}, \hat{v}(\hat{y}), \hat{I}),(4.3)$ implies

$$
\frac{d \hat{v}}{d \bar{y}}(\hat{y})=\frac{1}{r(\hat{\pi})} \frac{\hat{v}}{\hat{y}} .
$$


Upon combining (4.14), (4.15), and (4.16), one obtains $w(0)>0$, hence,

$$
\hat{\pi} \hat{y}-\hat{\pi} \bar{v}(\hat{y})-w(0)-\hat{I}+A<\hat{\pi} \hat{y}-\hat{\pi} \hat{v}-\hat{I}+A .
$$

However, by Lemma 3.4, $\hat{\pi} \hat{y}-\hat{\pi} \hat{v}-\hat{w}(0)-\hat{I}+A=\hat{\pi} \hat{y}-\hat{\pi} \hat{v}-\hat{I}+A=0$. With $w(0)>0$, therefore, the contract $(\hat{I}, \hat{\ell}, \hat{\pi}, \hat{y}, w(\cdot))$ violates the financiers' participation constraint.

\subsection{A Reassuring Result}

Distributions that satisfy (4.12) involve hazard rates that go to zero as $\theta$ goes out of bounds. They are thus quite special. Under more standard assumptions about the distribution of $\tilde{\theta}$, the problem discussed in Proposition 4.2 cannot arise. For a large class of cases, the presence of noise does not affect the outcome that can be reached under optimal contracting.

Proposition 4.3 Assume that $f$ takes the separable form (3.16) and that the effort cost function $\lambda$ satisfies

$$
\pi \lambda_{\pi \bar{y}}(\bar{y}, I, \pi)-\lambda_{\bar{y}}(\bar{y}, I, \pi) \geq 0
$$

for all $(\bar{y}, I, \pi)$ satisfying $\pi \lambda_{\pi}-\lambda>0$. Let $(\hat{I}, \hat{\ell}, \hat{\pi}, \hat{y}, \hat{w}(\cdot))$ be an optimal contract for the case $\tilde{\theta} \equiv 1$. If the distribution $F$ has a compact support or if $F$ satisfies

$$
\lim _{\rho \rightarrow \infty} \frac{E[\tilde{\theta} \mid \tilde{\theta} \geq \rho]}{\rho}=1,
$$

there exist constants $\alpha<1$ and $R>0$ such that, for $w(\cdot)$ given by

$$
w(y)=(1-\alpha) \max (y-R, 0),
$$

the contract $(\hat{I}, \hat{\ell}, \hat{\pi}, \hat{y}, w(\cdot))$ is an optimal contract when $\tilde{\theta}$ has the distribution $F$. The constants $R$ and $\alpha$ satisfy the equations

$$
\frac{E[\tilde{\theta} \mid \tilde{\theta} \geq R / \hat{y}]}{E[\tilde{\theta} \mid \tilde{\theta} \geq R / \hat{y}]-R / \hat{y}}=\frac{1}{r(\hat{\pi})}
$$

and

$$
1-\alpha=\frac{1}{r(\hat{\pi})} \frac{\hat{v}}{\hat{y}} \frac{1}{\int_{R / \hat{y}}^{\infty} \theta d F(\theta)}
$$


Before turning to the proof of this proposition, I make two remarks on its scope. First, if $f$ takes the separable homogeneous form (3.41), the additional assumption that is imposed in Proposition 4.3 is automatically satisfied if the elasticity of substitution between investment and effort is everywhere less than or equal to one. In this case,

$$
\pi \lambda_{\pi \bar{y}}-\lambda_{\bar{y}}=\left[\frac{\pi \lambda_{\pi}-\lambda}{\lambda}+\frac{r(\pi)}{\beta} \frac{\psi_{I} I}{\psi_{\ell} \ell} \frac{1-\sigma}{\sigma}\right] \lambda_{\bar{y}}
$$

and one has $\pi \lambda_{\pi \bar{y}}-\lambda_{\bar{y}} \geq 0$ if $\pi \lambda_{\pi}-\lambda>0$ and $\sigma \leq 1$. I do not know whether the conclusion of Proposition 4.3 might also hold if $\sigma$ were greater than one.

Second, if the distribution of $\tilde{\theta}$ has a density $\varphi$, then, by l'Hospital's rule, condition (4.19) is equivalent to the condition that $\lim _{\theta \rightarrow \infty} \frac{\theta \varphi(\theta)}{1-F(\theta)}=\infty$. This is necessarily satisfied if the hazard rate $\frac{\varphi}{1-F}$ is bounded away from zero.

The argument for Proposition 4.3 is illustrated in Figure 2, which uses a logarithmic scaling for both $\bar{y}$ and $\bar{v}$. With this double-logarithmic scaling, i.e., in $\ln \bar{y}$-ln $\bar{v}$-space, rather than $\bar{y}-\bar{v}$-space, Lemma 4.1 implies that the slope of the entrepreneur's indifference curve in $\ln \bar{y}$ - $\ln \bar{v}$-space is given by the elasticity $\frac{\bar{y}}{\hat{v}(\bar{y})} \frac{d \hat{v}}{d \bar{y}}(\bar{y})$. Under the additional assumptions on $f$ and $\lambda$, this elasticity is nondecreasing in $\bar{y}$; the entrepreneur's indifference curve in $\ln \bar{y}$ - $\ln \bar{v}$-space is convex and lies nowhere below a supporting tangent. In particular, the entrepreneur's indifference curve in $\ln \bar{y}$ - $\ln \bar{v}$-space lies nowhere below the straight line with slope $\frac{1}{r(\hat{\pi})}$ that goes through the point $(\hat{y}, \hat{v})=\left(\hat{y}, \lambda_{\pi}(\hat{y}, \hat{I}, \hat{\pi})\right)$. To prove the proposition, it is therefore sufficient to find an incentive scheme $w(\cdot) \in \mathcal{W}$ so that, as shown in the figure, pair $(\ln \bar{y}, \ln \bar{v}(\bar{y}))$ that is given by the induced $\bar{v}(\cdot)=\bar{w}(\cdot)-w(0)$ touches the straight line with slope $\frac{1}{r(\hat{\pi})}$ that goes through $(\hat{y}, \hat{v})$ in the point $(\hat{y}, \hat{v})$ itself and lies nowhere above it. The following lemma, whose proof is given in the Appendix, gives an additional condition on the distribution of $\tilde{\theta}$ under which this is possible.

Lemma 4.4 Let $\delta>1$ and suppose that the distribution $F$ has a compact support or that

$$
\frac{E[\tilde{\theta} \mid \tilde{\theta} \geq \rho]}{\rho}<\delta
$$

for any sufficiently large $\rho$. Then, for any $(\hat{y}, \hat{v}) \gg(0,0)$, there exist constants $R>0$ and $\alpha<1$ such that, for the incentive scheme $w(\cdot)$ given by (4.20), the induced functions $\bar{v}(\cdot)$ and $\bar{w}(\cdot)$ satisfy

$$
\bar{v}(\hat{y})=\bar{w}(\hat{y})=\hat{v}
$$




\section{FIGURE 2}

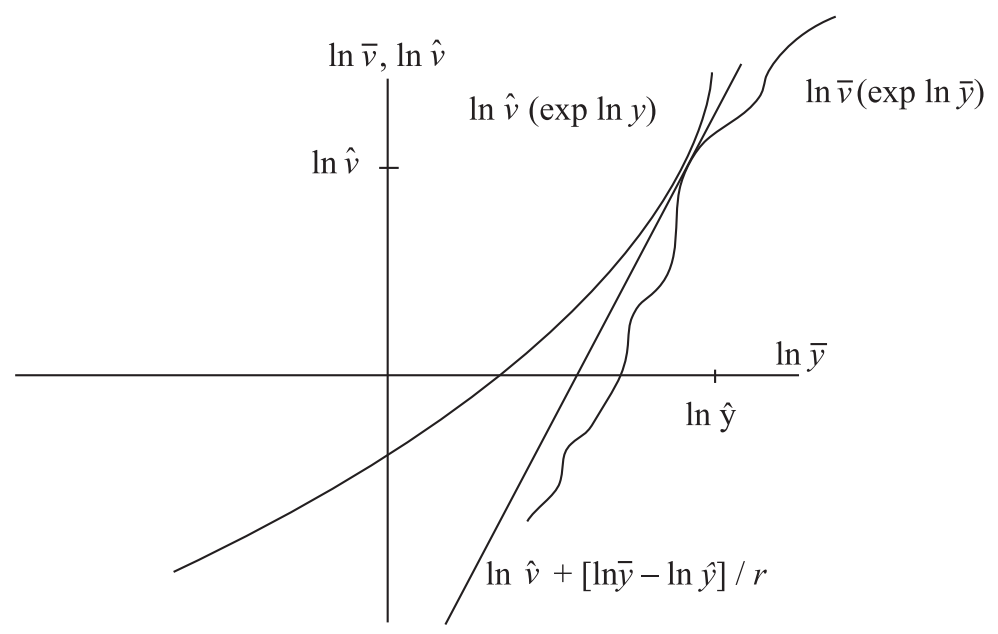

and, for all $\bar{y}>0$,

$$
\ln \bar{v}(\bar{y})=\ln \bar{w}(\bar{y}) \leq \ln \hat{v}+\delta(\ln \bar{y}-\ln \hat{y}) .
$$

The constants $R$ and $\alpha$ satisfy

$$
\frac{E[\tilde{\theta} \mid \tilde{\theta} \geq R / \hat{y}]}{E[\tilde{\theta} \mid \tilde{\theta} \geq R / \hat{y}]-R / \hat{y}}=\delta
$$

and

$$
1-\alpha=\frac{\hat{v}}{\hat{y}} \frac{\delta}{\int_{R / \hat{y}}^{\infty} \theta d F(\theta)} .
$$

Proof of Proposition 4.3. If the distribution $F$ has a compact support or if $F$ satisfies condition (4.19), then, for $\delta=\frac{1}{r(\hat{\pi})}$, the premise of Lemma 4.4 is satisfied. For the incentive scheme $w(\cdot)$ that this lemma yields for the given $\hat{y}, \hat{v}=\lambda_{\pi}(\hat{y}, \hat{I}, \hat{\pi})$, and $\delta=\frac{1}{r(\hat{\pi})}$, and for the induced $\bar{v}(\cdot)=\bar{w}(\cdot)-w(0)$, one obtains $\bar{v}(\hat{y})=\hat{v}$ and

$$
\ln \bar{v}(\bar{y}) \leq \ln \hat{v}+\frac{1}{r(\hat{\pi})}(\ln \bar{y}-\ln \hat{y})
$$


curve $\hat{v}(\cdot)$ through $\left(\hat{y}, \lambda_{\pi}(\hat{y}, \hat{I}, \hat{\pi})\right)$ is a nondecreasing function of $\bar{y}$, and one has

$$
\ln \hat{v}(\bar{y}) \geq \ln \hat{v}+\frac{1}{r(\hat{\pi})}(\ln \bar{y}-\ln \hat{y})
$$

for all $\bar{y}>0$. From (4.28) and (4.29), one obtains $\hat{v}(\bar{y}) \geq \bar{v}(\bar{y})$, hence, $U^{*}(\hat{y}, \hat{v}, \hat{I})=U^{*}(\bar{y}, \hat{v}(\bar{y}), \hat{I}) \geq U^{*}(\bar{y}, \bar{v}(\bar{y}), \hat{I})$ for all $\bar{y}>0$. The contract $(\hat{I}, \hat{\ell}, \hat{\pi}, \hat{y}, w(\cdot))$ thus satisfies the incentive constraint $(2.22)$ as well as the other constraints of the contracting problem. Given that this contract provides the entrepreneur with the same payoff as the contract $(\hat{I}, \hat{\ell}, \hat{\pi}, \hat{y}, \hat{w}(\cdot))$ that is optimal when the constraint (2.22) is not imposed, it follows that $(\hat{I}, \hat{\ell}, \hat{\pi}, \hat{y}, w(\cdot))$ is optimal when $\tilde{\theta}$ has the distribution $F$ and the constraint (2.22) has to be taken into account.

For an incentive scheme of the form (4.20), one computes

$$
\bar{w}(\bar{y})=(1-\alpha) \int_{R / \bar{y}}^{\infty}(\theta \bar{y}-R) d F(\theta)
$$

and

$$
\frac{\bar{y}}{\bar{w}(\bar{y})} \frac{d \bar{w}}{d \bar{y}}(\bar{y})=\frac{\int_{R / \bar{y}}^{\infty} \theta \bar{y} d F(\theta)}{\int_{R / \bar{y}}^{\infty}(\theta \bar{y}-R) d F(\theta)}=\frac{E[\tilde{\theta} \mid \tilde{\theta} \geq R / \bar{y}]}{E[\tilde{\theta} \mid \tilde{\theta} \geq R / \bar{y}]-R / \bar{y}} .
$$

Condition (4.26) thus fixes $R$ so that the elasticity of $\bar{w}(\cdot)$ at $\hat{y}$ is equal to the stipulated $\delta$; this corresponds to the requirement that, in the point $(\ln \hat{y}, \ln \hat{v})$ in Figure 2, the straight line with slope $\delta=\frac{1}{r(\hat{\pi})}$ is tangent to the graph of the function relating $\ln \bar{w}$ to $\ln \bar{y}$. Given $R$, condition (4.22) fixes $\alpha$ so that $\bar{w}(\hat{y})$ reaches the stipulated $\hat{v}$.

The additional assumption about the distribution of $\tilde{\theta}$ ensures that equation (4.21) actually has a solution; if $\frac{E[\tilde{\theta} \mid \tilde{\theta} \geq \rho]}{\rho}$ converges to one as $\rho$ becomes large, one can make the elasticity (4.31) as large as one wants simply by choosing $R$ to be sufficiently large. By contrast, for the distributions considered in Proposition 4.2, in the preceding subsection, one has $\frac{E[\tilde{\theta}[\tilde{\theta} \geq \rho]}{\rho}=\frac{k}{k-1}$ for $\rho \geq \bar{\theta}(k)$ so that the left-hand side of (4.21) is bounded above by $k$; for $\delta>k$, therefore, this equation has no solution. For these distributions, the requirement that the entrepreneur should be willing to choose the stipulated $\hat{y}$ over any $\bar{y} \neq \hat{y}$ can only be satisfied if $\frac{1}{r(\hat{\pi})} \leq k$. If the effort cost function satisfies the additional premises of Proposition 4.3, the inequality $\frac{1}{r(\hat{\pi})} \leq k$ is also sufficient to ensure that the requirement that the entrepreneur should be willing to choose the stipulated $\hat{y}$ over any $\bar{y} \neq \hat{y}$ can be satisfied. The 
presence of noise with such a distribution imposes the additional constraint that $\hat{\pi} \geq r^{-1}\left(\frac{1}{k}\right)$, i.e. that failure risk should not be "too great".

If the distribution of $\tilde{\theta}$ is such that, as $\rho$ becomes large, the ratio $\frac{E[\tilde{\theta} \mid \tilde{\theta} \geq \rho]}{\rho}$ decreases monotonically to a constant $C>1$, then, for an effort cost function with the stipulated properties, the requirement that the entrepreneur should be willing to choose the stipulated $\hat{y}$ over any $\bar{y} \neq \hat{y}$ can be satisfied if and only if $\hat{\pi}>r^{-1}\left(\frac{C-1}{C}\right)$. This condition defines an open set. Therefore, it may well be that, for noise having such a probability distribution, the contracting problem has a supremum, but not a maximum.

\section{Does the Jensen-Meckling Model Provide a The- ory of Debt and Equity Finance?}

To conclude the paper, I discuss the interpretation of the model as a model of debt and equity finance. Throughout the analysis, I have studied the model as a model of outside finance supported by a suitable incentive scheme $w(\cdot)$. I could have assumed that the incentive scheme must correspond to the residual claims that are left after debt and equity as the instruments of outside finance have been served. However, such an assumption would hardly be deemed to provide a proper basis for an "explanation" of the issue of debt and equity instruments as an incentive device.

For the case $\tilde{\theta}=1$, I have relied on forcing contracts, under which the financiers get the realization $y$ of the firm's return if this is less than the stipulated $\bar{y}$, and they get the difference $y-\bar{y}$ if $y$ is greater than $\bar{y}$. This arrangement could be interpreted as a wage contract with a wage payment conditioned on reaching the target $\bar{y}$; it cannot be interpreted as the result of some mix of debt and equity finance.

However, these forcing contracts are vulnerable to the introduction of noise. For the case of nondegenerate $\tilde{\theta}$, the contracts that were stipulated in Proposition 4.3 look as if they could be interpreted as the result of debt and equity finance. Under these contracts, the entrepreneur receives nothing if the return realization $y$ is less than $R$, and he receives a constant multiple $(1-\alpha)$ of any excess of $y$ over $R$. The parameter $R$ is positive and may be interpreted as a debt service obligation. The multiple $(1-\alpha)$ looks like the share that is retained when outside financiers hold a fraction $\alpha$ of the firm's equity.

The positivity of the parameter $R$ is a corollary of the result that an optimal contract involves excessive risk taking. This parameter is a solution to equation (4.21). Because $\hat{\pi}<\pi^{*}$ and, therefore, $r(\hat{\pi})<r\left(\pi^{*}\right)=1$, the 
right-hand side of this equation is greater than one. At $R<0$, the lefthand side of equation (4.21) is less than one, at $R=0$, it is equal to one. Any solution to equation (4.21) must therefore satisfy $R>0$. There is thus a direct link between the desirability of excessive risk taking in a secondbest contract and the observation of Jensen and Meckling that debt finance provides a way of reducing entrepreneur's share $\frac{\bar{w}(\bar{y})}{\bar{y}}$ of (conditionally) expected returns without reducing the slope $\frac{d \bar{w}}{d \bar{y}}$ and thereby weakening effort incentives.

The "share" parameter is more difficult to interpret. After substituting for $\hat{v}=\lambda_{\pi}(\hat{y}, \hat{I}, \hat{\pi})$, one can use (3.18) to rewrite (4.22) in the form

$$
1-\alpha=\frac{\lambda_{\bar{y}}(\hat{y}, \hat{I}, \hat{\pi})}{\hat{\pi}} \frac{1}{\int_{R / \hat{y}}^{\infty} \theta d F(\theta)} .
$$

The first-order condition (3.11) indicates that, under the assumptions of Proposition 4.3, one has $\hat{\pi}>\lambda_{\bar{y}}(\hat{y}, \hat{I}, \hat{\pi})$. Equation (5.32) therefore implies that $\alpha$ is positive if $\int_{R / \hat{y}}^{\infty} \theta d F(\theta)$ is close to one and negative if $\int_{R / \hat{y}}^{\infty} \theta d F(\theta)$ is close to zero. The following results show that, depending on the data of the model, both possibilities can actually arise.

Proposition 5.5 If the distribution of the random variables $\tilde{\theta}$ is such that, for some $\varepsilon \in(0, r(\hat{\pi}))$,

$$
F(1-\varepsilon)<\frac{1}{1-\varepsilon} \min \left(r(\hat{\pi})-\varepsilon, 1-\frac{\lambda_{\bar{y}}(\hat{y}, \hat{I}, \hat{\pi})}{\hat{\pi}}\right),
$$

and, moreover, the ratio $E[\tilde{\theta} \mid \tilde{\theta} \geq \rho] / \rho$ is a nonincreasing function of $\rho$, then the parameter $\alpha$ in Proposition 4.3 is positive, and the incentive scheme $w(\cdot)$ that is given by (4.20) can be interpreted as the result of giving outside financiers a debt service obligation $R>0$ and equity amounting to a share $\alpha$ in the firm.

Proof. By definition, $E[\tilde{\theta} \mid \tilde{\theta} \geq 1-\varepsilon] \leq \frac{1}{1-F(1-\varepsilon)}$. By (5.33), it follows that

$$
E[\tilde{\theta} \mid \tilde{\theta} \geq 1-\varepsilon] \leq \frac{1-\varepsilon}{1-\varepsilon-r(\hat{\pi})+\varepsilon}=\frac{1-\varepsilon}{1-r(\hat{\pi})}
$$

By the monotonicity assumption on $E[\tilde{\theta} \mid \tilde{\theta} \geq \rho] / \rho$, it follows that $E[\tilde{\theta} \mid \tilde{\theta} \geq$ $\rho] \leq \frac{\rho}{1-r(\hat{\pi})}$ for all $\rho \geq 1-\varepsilon$. Hence,

$$
\frac{E[\tilde{\theta} \mid \tilde{\theta} \geq \rho] / \rho}{E[\tilde{\theta} \mid \tilde{\theta} \geq \rho] / \rho-1}=\frac{E[\tilde{\theta} \mid \tilde{\theta} \geq \rho]}{E[\tilde{\theta} \mid \tilde{\theta} \geq \rho]-\rho}>\frac{\frac{1}{1-r(\hat{\pi})}}{\frac{1}{1-r(\hat{\pi})}-1}=\frac{1}{r(\hat{\pi})}
$$


for all $\rho \geq 1-\varepsilon$. Equation (4.21) therefore implies that $R / \hat{y}<1-\varepsilon$. Because the unconditional mean of $\tilde{\theta}$ is equal to one, one also has

$$
\int_{R / \hat{y}}^{\infty} \theta d F(\theta) \geq 1-F(R / \hat{y})>1-F(1-\varepsilon) .
$$

By (5.33), it follows that

$$
\int_{R / \hat{y}}^{\infty} \theta d F(\theta) \geq \frac{\lambda_{\bar{y}}(\hat{y}, \hat{I}, \hat{\pi})}{\hat{\pi}}
$$

and, therefore, that the right-hand side of (5.32) is less than one. Thus, $\alpha>0$.

Proposition 5.6 If the distribution of the random variables $\tilde{\theta}$ is such that, for some $\varepsilon \in(0,1-r(\hat{\pi})), F(1-r(\hat{\pi}))-F(\varepsilon)$ is close to one $e^{19}$, and, moreover, the ratio $E[\tilde{\theta} \mid \tilde{\theta} \geq \rho] / \rho$ is a nonincreasing function of $\rho$, then the parameter $\alpha$ in Proposition 4.3 is negative, and the incentive scheme $w(\cdot)$ that is given by (4.20) cannot be interpreted as the result of issuing a suitable mix of debt and equity to outside financiers.

Proof. I first claim that, under the assumptions of the proposition, one must have $\frac{R}{\hat{y}}>1-r(\hat{\pi})$. To establish this claim, I begin by noting that, on the support of the distribution $F$, the conditional expectation $E[\tilde{\theta} \mid \tilde{\theta} \geq \rho]$ is strictly increasing in $\rho$. Thus, $F(1-r(\hat{\pi}))>0$ implies $E[\tilde{\theta} \mid \tilde{\theta} \geq 1-r(\hat{\pi})]>1$. By the monotonicity assumption on the ratio $E[\tilde{\theta} \mid \tilde{\theta} \geq \rho] / \rho$, it follows that, for all $\rho \leq 1-r(\hat{\pi})$, one has

$$
\frac{E[\tilde{\theta} \mid \tilde{\theta} \geq \rho]}{\rho}>\frac{1}{1-r(\hat{\pi})},
$$

and, therefore, that $R=\rho \hat{y}$ cannot be a solution to equation (4.21).

Given that $\frac{R}{\hat{y}}>1-r(\hat{\pi})$, equation (5.32) implies

$$
1-\alpha>\frac{\lambda_{\bar{y}}(\hat{y}, \hat{I}, \hat{\pi})}{\hat{\pi}} \frac{1}{\int_{1-r(\hat{\pi})}^{\infty} \theta d F(\theta)} .
$$

\footnotetext{
${ }^{19}$ Using Chebyshev's inequality, one easily verifies that this is the case whenever the variance of $\tilde{\theta}$ is close to zero.
} 
To prove the proposition, it therefore suffices to show that, under the given assumptions, $\int_{1-r(\hat{\pi})}^{\infty} \theta d F(\theta)$ is close to zero. By the monotonicity assumption on $E[\tilde{\theta} \mid \tilde{\theta} \geq \rho] / \rho$, one has

$$
\begin{aligned}
\int_{1-r(\hat{\pi})}^{\infty} \theta d F(\theta) & \leq(1-r(\hat{\pi}))(1-F(1-r(\hat{\pi}))) \frac{E[\tilde{\theta} \mid \tilde{\theta} \geq \varepsilon]}{\varepsilon} \\
& \leq \frac{1-r(\hat{\pi})}{\varepsilon} \frac{1-F(1-r(\hat{\pi}))}{1-F(\varepsilon)} \\
& \leq \frac{1-r(\hat{\pi})}{\varepsilon}\left[1-\frac{F(1-r(\hat{\pi}))-F(\varepsilon)}{1-F(\varepsilon)}\right] \\
& \leq \frac{1-r(\hat{\pi})}{\varepsilon}[1-(F(1-r(\hat{\pi}))-F(\varepsilon))] .
\end{aligned}
$$

Hence, if $(F(1-r(\hat{\pi}))-F(\varepsilon))$ is close to one, $\int_{1-r(\hat{\pi})}^{\infty} \theta d F(\theta)$ is close to zero.

To understand these results, it is useful to rewrite equation (5.32) as

$$
(1-\alpha)\left[\int_{R / \hat{y}}^{\infty} \theta d F(\theta)\right] \hat{\pi}=\lambda_{\bar{y}}(\hat{y}, \hat{I}, \hat{\pi}),
$$

or

$$
(1-\alpha)\left[\int_{R / \hat{y}}^{\infty} \theta d F(\theta)\right] \hat{\pi} X(\hat{\pi}) g_{\ell}(\hat{I}, \hat{\ell})=1 .
$$

These conditions are just the first-order conditions for the entrepreneur's choice of $\bar{y}$ with effort cost $\lambda(\bar{y}, \hat{I}, \hat{\pi})$ or, equivalently, the entrepreneur's choice of $\ell$ when $\bar{y}=f(\hat{I}, \ell, \hat{\pi})$. The entrepreneur's share $1-\alpha$ in the firm's equity appears as an incentive device supporting the stipulated $\bar{y}=\hat{y}$ and $\ell=\lambda(\hat{y}, \hat{I}, \hat{\pi})$. In fixing this share, attention must be paid to the fact that the entrepreneur's incentives to work for an increase in $\bar{y}$ are blunted if the benefits of the increase do not accrue entirely to "equity holders". With $R>$ 0 , the "bankruptcy portion" $\int_{0}^{R / \hat{y}} \theta d F(\theta)$ of the total effect $\int_{0}^{1} \theta d F(\theta)=1$ of a unit increase in $\bar{y}$ accrues to "creditors" rather than "equity holders". To compensate for this effect, the entrepreneur's "equity share" $1-\alpha$ must be adjusted upwards. The amount of adjustment depends on the distribution of $\tilde{\theta}$. If the "default probability" is small, not much of an adjustment is needed, and, with $\hat{\pi}>\lambda_{\bar{y}}(\hat{y}, \hat{I}, \hat{\pi})$, one has $\alpha \in(0,1)$. In this case, the interpretation of Proposition 4.3 as a result about debt and equity finance is unproblematic. 
By contrast, if $\int_{R / \hat{y}}^{\infty} \theta d F(\theta)$ is close to zero, the entrepreneur's "equity share" $1-\alpha$ under the optimal contract in Proposition 4.3 must be very large. Thus, $1-\alpha$ must eventually exceed $100 \%$, at which point the financiers would have to "hold" negative equity. Alternatively, one could think of an arrangement under which the financiers hold $100 \%$ of the equity and the entrepreneur has a call option to buy an equity share $1-\alpha$ at the exercise price $R$ per unit. Under this arrangement, if $\int_{R / \hat{y}}^{\infty} \theta d F(\theta)$ is close to zero, the financiers' obligation under the call option held by the entrepreneur is strictly greater than the $100 \%$ equity that they hold. Under either arrangement, from a traditional finance perspective, the optimal contract in Proposition 4.3 seems somewhat outlandish.

One may object that, for $\int_{R / \hat{y}}^{\infty} \theta d F(\theta)$ close to zero, the contract in Proposition 4.3 cannot be taken seriously because the analysis has not taken account of possible limitations on the financiers' ability or willingness to provide the entrepreneur with a return $w(\tilde{y})$ above the firm return $\tilde{y}$. This objection is valid and important, but it is somewhat besides the point under discussion here. By imposing the additional constraint that $w(y) \leq y$ for all $y$, one rules out incentive schemes of the form (4.20) with $\alpha<0$, but one does not eliminate the desire to raise the sensitivity of $w(y)$ to an increase in $y$ in order to compensate for the blunting of incentives that is due to the noise. The analysis of Innes (1990) or Dionne and Viala (1992) suggests that such considerations should lead to contracts with discontinuous incentive schemes of the form $w(y)=0$ for $y<y^{*}$ and $w(y)=y$ for $y \geq y^{*}$, for some $y^{*}$, the jump at $y^{*}$ making an extra contribution to the slope of the function $\bar{w}(\cdot)$. This would seem to lead us even farther from the world of traditional finance contracts and into the world of abstract incentive schemes.

Innes (1990) proposes to eliminate this effect by imposing the additional requirement that $y-w(y)$ must be nondecreasing in $y$. I suspect that in the context of Proposition 5.6, as in Innes's own analysis, this constraint would lead to the optimality of pure debt finance, i.e. an incentive scheme of the form (4.20) with $\alpha=0$, inducing a lower $\bar{y}$ and a higher $\pi$. However, the monotonicity requirement on $y-w(y)$ is problematic. To support this requirement, Innes invokes a free-disposal option for financiers or, alternatively, an option that the entrepreneur has of faking a higher return realization by borrowing the shortfall from a third party, thereby winning a yet higher increase in his own payoff, part of which serves to repay the amount borrowed. Both these arguments are moot if returns are observable and verifiable.

Doubts about the relation between incentive contracting and financial 
packaging must run even deeper. Even the rather comforting conclusion of Proposition 5.5 is due to a certain arbitrariness of optimal incentive schemes rather than any substantive virtues of piecewise linearity of incentive schemes, let alone debt and equity instruments. Using the same arguments as in the proof of Proposition 4.3, one can in fact show that the conclusion of that proposition remains true if the piecewise linear scheme (4.20) is replaced by a piecewise quadratic scheme of the form

$$
w(y)=(1-\alpha) \max \left[y-R-\beta(y-R)^{2}, 0\right],
$$

where $\beta$ is an arbitrary small positive number. The reason is that, when all parties are risk neutral, the precise form of the incentive scheme does not matter provided that, in expected-value terms, it provides the requisite payoffs and incentives. ${ }^{20}$

\footnotetext{
${ }^{20}$ Along the lines of Innes's arguments, the reader may object that, for $y-R \in\left[\frac{1}{2 \beta}, \frac{1}{\beta}\right]$, the scheme (5.36) decreasing in $y$, which is not incentive-compatible if the entrepreneur can freely destroy output. The argument is valid, but hardly matters if noise is small enough so that the return realization $\tilde{\theta} \hat{y}$ does not actually reach the region of nonmonotonicity. If, instead, the range of $\tilde{\theta}$ is all of $\mathbb{R}_{+}$, the basic point remains valid if (5.36) is modified so that, for $y \geq R+\frac{1}{2 \beta}, w(y)$ remains constant at $(1-\alpha) \frac{1}{4 \beta}$.
} 


\section{A Appendix}

Proof of Lemma 2.1. I first show that an optimal contract satisfies $\pi \bar{y}>0$. For this purpose, it suffices to show that there exists a contract that dominates the best available contract with $\pi \bar{y}=0$. Trivially, the entrepreneur's expected payoff from the best available contract with $\pi \bar{y}=0$ is equal to $A$, the amount he can earn by investing his initial holdings in the market. I will show that, under the given assumptions on $f$, there exists a contract that provides him a payoff greater than $A$.

It suffices to consider contracts that involve simple sharing rules $w_{\alpha}(\cdot)$, where, for any $y$,

$$
w_{\alpha}(y)=A+(1-\alpha) y .
$$

Under such a sharing rule, expected payoffs are

$$
A+(1-\alpha) \pi f(I, \ell, \pi)-\ell
$$

for the entrepreneur and

$$
\alpha \pi f(I, \ell, \pi)-I
$$

for the financiers. For any $\alpha \in(0,1)$ and $I \geq 0$, let $\psi(\alpha, I)$ be the set of pairs $(\ell, \pi)$ that maximize the entrepreneur's expected payoff (A.2), and let $(\ell(\alpha, I), \pi(\alpha, I)) \in \psi(\alpha, I)$. Then the contract $C(\alpha, I)$, which stipulates the return target $\bar{y}(\alpha, I)=f(I, \ell(\alpha, I), \pi(\alpha, I))$, the investment level $I$, then entrepreneur's choice $(\ell(\alpha, I), \pi(\alpha, I))$ and the incentive scheme $w_{\alpha}(\cdot)$, is incentive-compatible.

By construction, the entrepreneur's payoff from this contract satisfies

$$
\begin{aligned}
A+(1-\alpha) \pi f(I, \ell, \pi)-\ell & \geq A+(1-\alpha) \pi_{0} f\left(I, \lambda_{0} I, \pi_{0}\right)-\lambda_{0} I \\
& \geq A+I\left[(1-\alpha) \pi_{0} \frac{f\left(I, \lambda_{0} I, \pi_{0}\right)}{I}-\lambda_{0}\right]
\end{aligned}
$$

where $\lambda_{0}, \pi_{0}$ are as given in the Inada conditions. By equation (2.27) in the text, it follows that the entrepreneur's payoff from the contract $C(\alpha, I)$ is strictly greater than $A$ if $I$ is sufficiently close to zero. fies

By the same logic, the financiers' payoff from the contract $C(\alpha, I)$ satis-

$$
\begin{aligned}
\alpha \pi f(I, \ell, \pi)-I & \geq \frac{\alpha}{1-\alpha}[(1-\alpha) \pi f(I, \ell, \pi)-\ell]-I \\
& \geq \frac{\alpha}{1-\alpha}\left[(1-\alpha) \pi_{0} f\left(I, \lambda_{0} I, \pi_{0}\right)-\lambda_{0} I\right]-I \\
& =I\left[\alpha \pi_{0} \frac{f\left(I, \lambda_{0} I, \pi_{0}\right)}{I}-\frac{\alpha}{1-\alpha} \lambda_{0}-1\right] .
\end{aligned}
$$


By equation (2.27) in the text, the right-hand side of (A.5) is strictly positive if $I$ is sufficiently close to zero.

For $\alpha \in(0,1)$ and $I>0$ sufficiently close to zero, the contract $C(\alpha, I)$ thus satisfies the financiers' participation constraint, as well as being incentivecompatible and providing the entrepreneur with a payoff greater than $A$. Therefore, an optimal contract must provide the entrepreneur with a payoff greater than $A$. Therefore, an optimal contract satisfies $\pi \bar{y}>0$. Trivially, this implies $\bar{y}>0$ and $\pi>0$. By (2.24), one must also have $\pi<1$.

By (2.24), one must also have $I>0$ or $\ell>0$. It remains to be shown that both these inequalities hold. Suppose first that $I=0$. Then one must have $w(\cdot)=w_{0}(\cdot), \ell=\ell(0,0)>0$, and $\pi=\pi(0,0)>0$. The entrepreneur's expected payoff must be

$$
A+\pi(0,0) \bar{y}(0,0)-\ell(0,0)>A .
$$

The optimality of the contract implies

$$
\pi(0,0) \bar{y}(0,0)-\ell(0,0) \geq(1-\alpha) \pi(\alpha, I) \bar{y}(\alpha, I)-\ell(\alpha, I)
$$

for all $\alpha, I$ for which

$$
\alpha \pi(\alpha, I) \bar{y}(\alpha, I) \geq I \text {. }
$$

Because, for any $\alpha$ and $I$, the pair $(\ell(\alpha, I), \pi(\alpha, I))$ maximizes (A.2), it is easy to see that the map $(\alpha, I) \rightarrow \pi(\alpha, I) \bar{y}(\alpha, I)$ is nonincreasing in $\alpha$ and nondecreasing in $I$. Suppose that, for any $\alpha$ and $I,(\ell(\alpha, I), \pi(\alpha, I))$ is actually chosen so that the product $\pi(\alpha, I) f(I, \ell(\alpha, I), \pi(\alpha, I))$ is minimal in $\psi(\alpha, I)$. Then, by the maximum theorem, the function $\alpha \rightarrow \pi(\alpha, 0) \bar{y}(\alpha, 0)$ is continuous from the right. In particular, for any $\varepsilon>0$, there exists $\delta>0$ such that, for $\alpha \in[0, \delta)$, one has

$$
\pi(\alpha, 0) \bar{y}(\alpha, 0) \geq \frac{1}{1+\varepsilon} \pi(0,0) \bar{y}(0,0) .
$$

By the monotonicity of $\pi(\alpha, I) \bar{y}(\alpha, I)$ in $I, \alpha \in[0, \delta)$ also implies

$$
\pi(\alpha, I) \bar{y}(\alpha, I) \geq \frac{1}{1+\varepsilon} \pi(0,0) \bar{y}(0,0)
$$

for all $I$. The financiers' participation constraint (A.8) is therefore satisfied whenever $I$ is sufficiently small so that, for

$$
\alpha=\frac{(1+\varepsilon) I}{\pi(0,0) \bar{y}(0,0)}
$$


ane has $\delta \geq \alpha$. For such $\alpha$ and $I$, revealed preference implies

$$
(1-\alpha) \pi(\alpha, I) \bar{y}(\alpha, I)-\ell(\alpha, I) \geq(1-\alpha) \pi(0,0) f(I, \ell(0,0), \pi(0,0))-\ell(0,0) .
$$

By the mean value theorem, it follows that, for some $I^{\prime} \in[0, I]$, one has

$$
\begin{aligned}
(1-\alpha) \pi(\alpha, I) \bar{y}(\alpha, I)-\ell(\alpha, I) \geq & \pi(0,0) \bar{y}(0,0)-\ell(0,0) \\
& +\pi(0,0) f_{I}\left(I^{\prime}, \ell(0,0), \pi(0,0)\right) I \\
& -\alpha \pi(0,0) f(I, \ell(0,0), \pi(0,0)) .
\end{aligned}
$$

By (A.11), the last two terms on the right-hand side can be rewritten as

$$
I\left[\pi(0,0) f_{I}\left(I^{\prime}, \ell(0,0), \pi(0,0)\right)-(1+\varepsilon) \frac{f(I, \ell(0,0), \pi(0,0))}{f(0, \ell(0,0), \pi(0,0))}\right] .
$$

By the Inada condition (2.26) for $f_{I}$, this expression is strictly positive if $I$ and, therefore, $I^{\prime}$ are close to zero. Therefore, (A.12) implies

$$
(1-\alpha) \pi(\alpha, I) \bar{y}(\alpha, I)-\ell(\alpha, I)>\pi(0,0) \bar{y}(0,0)-\ell(0,0)
$$

if $I$ is close to zero. The assumption that $I=0$ has thus led to a contradiction and must be false.

Finally, suppose that $\ell=0$. Because of the Inada condition (2.26) for $f_{\ell}$, incentive compatibility implies that $\bar{w}(\bar{y})=w(0)$. The incentive scheme may therefore be taken to be constant. Given the financiers' participation constraint, it follows that $w(y)=A+\pi \bar{y}-I$, regardless of $y$. The entrepreneur's expected payoff from the optimal contract is also equal to $A+\pi \bar{y}-I$ By the argument given above, this must be greater than $A$, i.e. one must have $I<\pi \bar{y}$.

Consider the contract $C(\alpha, I)$ that is given by the sharing rule $w_{\alpha}(\cdot)$, with $\alpha=\frac{I}{\pi \bar{y}}$, and the investment $I$. Because $I<\pi \bar{y}$, one has $\alpha<1$. By the monotonicity of the map $\alpha \rightarrow \pi(\alpha, I) \bar{y}(\alpha, I)$, the financiers' payoff from the contract $C(\alpha, I)$ satisfies

$$
\alpha \pi(\alpha, I) \bar{y}(\alpha, I)-I \geq \alpha \pi \bar{y}-I=0,
$$

which shows that their participation constraint is satisfied. Because of the Inada condition (2.26) for $f_{\ell}, \alpha<1$ also implies that the pair $(\ell(\alpha, I), \pi(\alpha, I))$ is strictly better for the entrepreneur than the pair $(0, \pi)$. Therefore, his payoff from the contract $C(\alpha, I)$ satisfies

$$
A+(1-\alpha) \pi(\alpha, I) \bar{y}(\alpha, I)-\ell(\alpha, I)>A+(1-\alpha) \pi \bar{y}
$$


By construction, the right-hand side is equal to $A+\pi \bar{y}-I$. The entrepreneur's payoff from the contract $C(\alpha, I)$ is thus strictly greater than his payoff from the optimal contract. The assumption that $\ell=0$ has thus led to a contradiction and must be false.

Proof of Proposition 3.10. Using (3.44) and (3.45), one can rewrite (3.48) as

$$
\begin{aligned}
-1+\frac{\psi_{\ell}}{\psi_{I}} & =\frac{\mu-1}{\mu}\left[\frac{1}{\sigma} \frac{r(\pi)}{\beta} \frac{\psi}{\psi_{\ell} \ell}-1\right] \\
& =\frac{\mu-1}{\mu}\left[\frac{1}{\sigma} \frac{r(\pi)}{\beta}\left(1+\frac{\psi_{I} I}{\psi_{\ell} \ell}\right)-1\right] .
\end{aligned}
$$

If $\psi$ is given as

$$
\psi(I, \ell)=\left[a^{1-\alpha} I^{\alpha}+(1-a)^{1-\alpha} \ell^{\alpha}\right]^{\frac{1}{\alpha}}
$$

one has

$$
\frac{\psi_{\ell}}{\psi_{I}}=\left[\frac{(1-a) I}{a \ell}\right]^{1-\alpha} .
$$

The first-order conditions for $I^{*}$ and $\ell^{*}$ imply $\psi_{\ell}\left(I^{*}, \ell^{*}\right)=\psi_{I}\left(I^{*}, \ell^{*}\right)$. For the CES specification (A.16), one therefore has

$$
\frac{I^{*}}{\ell^{*}}=\frac{a}{1-a} .
$$

It is convenient to introduce new variable

$$
\eta:=\frac{I}{\ell} \frac{\ell^{*}}{I^{*}}=\frac{(1-a) I}{a \ell},
$$

so that (A.17) becomes $\frac{\psi_{\ell}}{\psi_{I}}=\eta^{1-\alpha}$, and (A.15) can be rewritten as

$$
\eta^{1-\alpha}-1=\frac{\mu-1}{\mu}\left[\frac{1}{\sigma} \frac{r(\pi)}{\beta}\left(1+\frac{a}{1-a} \eta^{\alpha}\right)-1\right] .
$$

The proposition is equivalent to the claim that any solution to this equation must satisfy $\eta<1$ if $\sigma=\frac{1}{1-\alpha}$ is sufficiently large.

To prove this claim, I first observe that, for $\eta=0$, the left-hand side of this equation is -1 and the right-hand side is greater than $-\frac{\mu-1}{\mu}>-1$, i.e., the left-hand side is smaller than the right-hand side. For $\eta=1$, the left-hand side is equal to zero. As for the right-hand side, Proposition 3.5 and (3.46) imply that $r(\pi)<1$. For $\eta=1$, the right-hand side of (A.20) is therefore equal to $\frac{\mu-1}{\mu}$ times a factor that is less than $\frac{1}{\sigma \beta(1-a)}-1$, which is 
negative if $\sigma>\frac{1}{\beta(1-a)}$. For $\sigma>\frac{1}{\beta(1-a)}$, therefore, the left-hand side of (A.20) is greater than the right-hand side. Equation (A.20) then has a solution that lies between zero and one.

If $\sigma>2$ or, equivalently, $\alpha>\frac{1}{2}$, the solution is in fact unique. To see this, it suffices to note that the difference between the left-hand side and the right-hand side of (A.20) has a derivative with respect to $\eta$ that is equal to

$$
(1-\alpha) \eta^{-\alpha}-\frac{\mu-1}{\mu} \frac{1}{\sigma} \frac{r(\pi)}{\beta} \frac{a}{1-a} \alpha \eta^{\alpha-1}=(1-\alpha) \eta^{-\alpha}\left[1-\frac{\mu-1}{\mu} \frac{r(\pi)}{\beta} \frac{a}{1-a} \alpha \eta^{2 \alpha-1}\right] .
$$

If $\alpha>\frac{1}{2}$, the term in brackets is decreasing in $\eta$, i.e., if this term is negative for some $\eta$, it must also be negative for all $\eta^{\prime}>\eta$. Given that the difference between the left-hand side and the right-hand side of (A.20) is positive for $\eta=0$ and zero at a solution to (A.20), it follows that this difference must be decreasing and therefore be negative at all higher values of $\eta$. It follows that, for $\sigma>2$, the solution to (A.20) is unique. If, in addition, $\sigma>\frac{1}{\beta a}$, the solution satisfies $\eta<1$, which is just (3.56).

Proof of Lemma 4.4. Given the distribution $F$, define a left-continuous and nondecreasing function $\hat{\theta}_{F}(\cdot)$ by setting

$$
\hat{\theta}_{F}(\rho)=E[\tilde{\theta} \mid \tilde{\theta} \geq \rho] \text { if } F(\rho)<1
$$

and

$$
\hat{\theta}_{F}(\rho)=\rho \text { if } F(\rho)=1 .
$$

If the support of $F$ is compact or if $F$ satisfies condition (4.23), then for the given $\delta>1$, one has

$$
\frac{\hat{\theta}_{F}(\rho)}{\rho}<\delta
$$

for any sufficiently large $\rho$. I further define a function $\chi(\cdot)$ by setting

$$
\chi(z)=\delta z-\int_{0}^{z} \frac{\hat{\theta}_{F}\left(e^{t}\right)}{\hat{\theta}_{F}\left(e^{t}\right)-e^{t}} d t .
$$

By construction, $F\left(e^{z}\right)<1$ implies that, for $t \leq z, \hat{\theta}_{F}\left(e^{t}\right)-e^{t}$ is bounded away from zero. Therefore, $\chi(z)$ is well defined.

I claim that the function $\chi(\cdot)$ has a global maximum at some $z^{*}$ satisfying

$$
\frac{\hat{\theta}_{F}\left(e^{z^{*}}\right)}{\hat{\theta}_{F}\left(e^{z^{*}}\right)-e^{z^{*}}}=\delta .
$$


To establish this claim, I first show that, at any $z<\ln \left(1-\frac{1}{\delta}\right)$, the function $\chi(\cdot)$ is strictly increasing. For this purpose, I note that, by the assumption that $E \tilde{\theta}=1$, one has $\hat{\theta}_{F}(0)=\int_{0}^{\infty} \theta d F(\theta)=1$. Because the function $\hat{\theta}_{F}(\cdot)$ is nondecreasing, it follows that $\hat{\theta}_{F}\left(e^{t}\right) \geq 1$ for all $t$. For $z<\ln \left(1-\frac{1}{\delta}\right)$, it follows that

$$
\chi^{\prime}(z)=\delta-\frac{\hat{\theta}_{F}\left(e^{z}\right)}{\hat{\theta}_{F}\left(e^{z}\right)-e^{z}} \geq \delta-\frac{1}{1-e^{z}}>0 .
$$

(If $\hat{\theta}_{F}(\cdot)$ is discontinuous at $e^{z}$, the inequality still holds for the one-sided derivatives of $\chi(\cdot)$.)

I next note that, by (A.23), there exists $\bar{z}$ such that, for all $z>\bar{z}$, one has

$$
\frac{\hat{\theta}_{F}\left(e^{z}\right)}{\hat{\theta}_{F}\left(e^{z}\right)-e^{z}}=\frac{\frac{\hat{\theta}_{F}\left(e^{z}\right)}{e^{z}}}{\frac{\hat{\theta}_{F}\left(e^{z}\right)}{e^{z}}-1}>\frac{\delta}{\delta-1}>\delta
$$

and, therefore,

$$
\chi^{\prime}(z)=\delta-\frac{\hat{\theta}_{F}\left(e^{z}\right)}{\hat{\theta}_{F}\left(e^{z}\right)-e^{z}}<0 .
$$

Thus, at any $z>\bar{z}$, the function $\chi(\cdot)$ is nonincreasing.

Because the function $\chi(\cdot)$ is obviously continuous on its domain, the restriction of this function to the compact interval $\left[\ln \left(1-\frac{1}{\delta}\right), \bar{z}\right]$ has a maximum, say at $z^{*} \in\left[\ln \left(1-\frac{1}{\delta}\right), \bar{z}\right]$. Because $\chi(\cdot)$ is increasing at any $z<\ln \left(1-\frac{1}{\delta}\right)$ and nonincreasing at any $z>\bar{z}, z^{*}$ is in fact a global maximizer of $\chi(\cdot)$. Thus, one has

$$
\int_{z}^{z^{*}} \frac{\hat{\theta}_{F}\left(e^{t}\right)}{\hat{\theta}_{F}\left(e^{t}\right)-e^{t}} d t \leq \delta\left(z^{*}-z\right)
$$

for all $z \in \mathbb{R}$.

Upon taking limits in (A.26) as $z$ converges to $z^{*}$ from below, using the left-contunity of the function $\hat{\theta}_{F}(\cdot)$, one has

$$
\frac{\hat{\theta}_{F}\left(e^{z^{*}}\right)}{\hat{\theta}_{F}\left(e^{z^{*}}\right)-e^{z^{*}}}=\lim _{t \uparrow z^{*}} \frac{\hat{\theta}_{F}\left(e^{t}\right)}{\hat{\theta}_{F}\left(e^{t}\right)-e^{t}} \leq \delta .
$$

Similarly, taking limits in (A.26) as $z$ converges to $z^{*}$ from above, one obtains

$$
\lim _{z \downarrow z^{*}} \sup _{t \in\left(z^{*}, z\right)} \frac{\hat{\theta}_{F}\left(e^{t}\right)}{\hat{\theta}_{F}\left(e^{t}\right)-e^{t}} \geq \delta
$$


Because $\hat{\theta}_{F}(\cdot)$ is nondecreasing, one also has

$$
\frac{\hat{\theta}_{F}\left(e^{z^{*}}\right)}{\hat{\theta}_{F}\left(e^{z^{*}}\right)-e^{z^{*}}} \geq \lim _{z \downarrow z^{*}} \sup _{t \in\left(z^{*}, z\right)} \frac{\hat{\theta}_{F}\left(e^{t}\right)}{\hat{\theta}_{F}\left(e^{t}\right)-e^{t}} .
$$

From (A.27) - (A.29), one obtains (A.25).

From (A.25), one also obtains $\hat{\theta}_{F}\left(e^{z^{*}}\right)>e^{z^{*}}$ and $F\left(e^{z^{*}}-\right)<1$. Therefore, one can define

$$
R=\hat{y} e^{z^{*}}
$$

and

$$
\alpha=1-\frac{\hat{v}}{\hat{y}} \frac{1}{\left(\hat{\theta}_{F}\left(e^{z^{*}}\right)-e^{z^{*}}\right)\left(1-F\left(e^{z^{*}}-\right)\right)} .
$$

For the incentive scheme $w(\cdot)$ that is defined by $w(y)=(1-\alpha) \max (y-R, 0)$, one obtains

$$
\begin{aligned}
\bar{w}(\bar{y}) & =\int_{0}^{\infty}(1-\alpha) \max (\theta \bar{y}-R, 0) d F(\theta) \\
& =\frac{\hat{v}}{\hat{y}} \frac{\int_{R / \bar{y}-}^{\infty}(\theta \bar{y}-R) d F(\theta)}{\int_{e^{z^{*}-}}^{\infty}\left(\theta-e^{z^{*}}\right) d F(\theta)}=\hat{v} \frac{\int_{R / \bar{y}-}^{\infty}(\theta \bar{y}-R) d F(\theta)}{\int_{R / \hat{y}-}^{\infty}(\theta \hat{y}-R) d F(\theta)}
\end{aligned}
$$

Clearly, $\bar{w}(\hat{y})=\hat{v}$. Moreover,

$$
\begin{aligned}
\frac{d \bar{w}}{d \bar{y}}(\bar{y}) & =\hat{v} \frac{\int_{R / \bar{y}-}^{\infty} \theta d F(\theta)}{\int_{R / \hat{y}-}^{\infty}(\theta \hat{y}-R) d F(\theta)}=\frac{\bar{w}(\bar{y})}{\bar{y}} \frac{\int_{R / \bar{y}-}^{\infty} \theta d F(\theta)}{\int_{R / \bar{y}-}^{\infty}\left(\theta-\frac{R}{\bar{y}}\right) d F(\theta)} \\
& =\frac{\bar{w}(\bar{y})}{\bar{y}} \frac{\hat{\theta}_{F}\left(\frac{R}{\bar{y}}\right)}{\hat{\theta}_{F}\left(\frac{R}{\bar{y}}\right)-\frac{R}{\bar{y}}}=\frac{\bar{w}(\bar{y})}{\bar{y}} \frac{\hat{\theta}_{F}\left(e^{z^{*}+\ln \hat{y}-\ln \bar{y}}\right)}{\hat{\theta}_{F}\left(e^{z^{*}+\ln \hat{y}-\ln \bar{y}}\right)-e^{z^{*}+\ln \hat{y}-\ln \bar{y}}} .
\end{aligned}
$$

For the function $s \rightarrow \gamma(s):=\ln \bar{w}\left(e^{s}\right)$, one therefore finds

$$
\frac{d \gamma}{d s}=\frac{e^{s}}{\bar{w}\left(e^{s}\right)} \frac{d \bar{w}}{d \bar{y}}\left(e^{s}\right)=\frac{\hat{\theta}_{F}\left(e^{z^{*}+\ln \hat{y}-s}\right)}{\hat{\theta}_{F}\left(e^{z^{*}+\ln \hat{y}-s}\right)-e^{z^{*}+\ln \hat{y}-s}}
$$

and

$$
\begin{aligned}
\gamma(\ln \bar{y})-\gamma(\ln \hat{y}) & =\int_{\ln \hat{y}}^{\ln \bar{y}} \frac{\hat{\theta}_{F}\left(e^{z^{*}+\ln \hat{y}-s}\right)}{\hat{\theta}_{F}\left(e^{z^{*}+\ln \hat{y}-s}\right)-e^{z^{*}+\ln \hat{y}-s}} d s \\
& =\int_{z^{*}+\ln \hat{y}-\ln \bar{y}}^{z^{*}} \frac{\hat{\theta}_{F}\left(e^{t}\right)}{\hat{\theta}_{F}\left(e^{t}\right)-e^{t}} d t
\end{aligned}
$$


the last equation following by the change of variables $t=z^{*}+\ln \hat{y}-s$. By (A.26), it follows that

$$
\ln \bar{w}(\bar{y})-\ln \bar{w}(\hat{y})=\gamma(\ln \bar{y})-\gamma(\ln \hat{y}) \leq \delta(\ln \bar{y}-\ln \hat{y})
$$

for all $\bar{y}$. 


\section{References}

[1] Aghion, P., and P. Bolton (1989), The Financial Structure of the Firm and the Problem of Control, European Economic Review 33, 186 - 293.

[2] Aghion, P., and P. Bolton (1992), An Incomplete-Contracting Approach to Financial Contracting, Review of Economic Studies 59, 473 - 494.

[3] Bester, H., and M.F. Hellwig (1987), Moral Hazard and Equilibrium Credit Rationing: An Overview of the Issues, in: G. Bamberg and K. Spreemann (eds.), Agency Theory, Information, and Incentives, Springer Verlag, Berlin, 135 - 166.

[4] Bolton, P., and M. Dewatripont, Contract Theory, MIT Press, Cambridge, Massachusetts.

[5] Caillaud, B., R. Guesnerie, and P. Rey (1992), Noisy Observation in Adverse Selection Models, Review of Economic Studies 59, 595 - 615.

[6] Dewatripont, M., and J. Tirole (1994), A Theory of Debt and Equity: Diversity of Securities and Manager-Shareholder Congruence, Quarterly Journal of Economics

[7] Dionne, G., and P. Viala (1992), Optimal Design of Financial Contracts and Moral Hazard, Cahier 9219, Université der Montréal.

[8] Gale, D.M., and M.F. Hellwig (1985), Incentive-Compatible Debt Contracts: The One-Period Problem, Review of Economic Studies 52, 647 -664 .

[9] Grossman, S.J., and O.D. Hart (1983), An Analysis of the PrincipalAgent Problem, Econometrica 51, 7 - 45.

[10] Harris, M., and A. Raviv (1991), The Theory of Capital Structure, Journal of Finance 46, 297 - 355.

[11] Hart, O.D. (1993), Theories of Optimal Capital Structure: A Managerial Discretion Perspective, in: M.M. Blair (ed.), The Deal Decade: What Takeovers and Leveraged Buyouts Mean for Corporate Governance, Brookings, Washington, D.C., 19 - 53.

[12] Hart, O.D. (1995), Firms, Contracts, and Financial Structure, Oxford University Press. 
[13] Hellwig, M.F. (1994), A Reconsideration of the Jensen-Meckling Model of Outside Finance, WWZ Discussion Paper No. 9416, University of Basel, Switzerland.

[14] Holmström, B. (1979), Moral Hazard and Observability, Bell Journal of Economics 10, 74 - 91.

[15] Holmström, B., and P. Milgrom (1987), Aggregation and Linearity in the Provision of Intertemporal Incentives, Econometrica 55, 303 - 328.

[16] Innes, R.D. (1990), Limited Liability and Incentive Contracting with Ex-Ante Action Choices, Journal of Economic Theory 52 ,

[17] Jensen, M.J., and W.R. Meckling (1976), Theory of the Firm: Managerial Behavior, Agency Cost, and Ownership Structure, Journal of Financial Economics 3, 305 - 360.

[18] Shavell, S. (1979), Risk Sharing and Incentives in the Principal and Agent Relationship, Bell Journal of Economics 10, 55 -73.

[19] Stiglitz, J.E., and A.W. Weiss (1981), Credit Rationing with Imperfect Information, American Economic Review 71, 393 - 410.

[20] Townsend, R. (1979), Optimal Contracts and Competitive Markets with Costly State Verification, Journal of Economic Theory 21, 417 -425 . 